Check for updates

Cite this: Nanoscale Adv., 2019, 1, 4207

\title{
Hydrophobic ion pairing: encapsulating small molecules, peptides, and proteins into nanocarriers
}

\author{
Kurt D. Ristroph and Robert K. Prud'homme (D)*
}

Hydrophobic ion pairing has emerged as a method to modulate the solubility of charged hydrophilic molecules ranging in class from small molecules to large enzymes. Charged hydrophilic molecules are ionically paired with oppositely-charged molecules that include hydrophobic moieties; the resulting uncharged complex is water-insoluble and will precipitate in aqueous media. Here we review one of the most prominent applications of hydrophobic ion pairing: efficient encapsulation of charged hydrophilic molecules into nano-scale delivery vehicles - nanoparticles or nanocarriers. Hydrophobic complexes are formed and then encapsulated using techniques developed for poorly-water-soluble therapeutics. With this approach, researchers have reported encapsulation efficiencies up to $100 \%$ and drug loadings up to $30 \%$. This review covers the fundamentals of hydrophobic ion pairing, including nomenclature, drug eligibility for the technique, commonly-used counterions, and drug release of encapsulated ion paired complexes. We then focus on nanoformulation techniques used in concert with hydrophobic ion pairing and note strengths and weaknesses specific to each. The penultimate section bridges hydrophobic ion pairing with the related fields of polyelectrolyte coacervation and polyelectrolyte-surfactant complexation. We then discuss the state of the art and anticipated future challenges. The review ends with comprehensive tables of reported hydrophobic ion pairing and encapsulation from the literature.

Received 16th May 2019

Accepted 18th September 2019

DOI: 10.1039/c9na00308h

rsc.li/nanoscale-advances section bridges the HIP technique with polyelectrolyte-polyelectrolyte complexation ('coacervation') and polyelectrolytesurfactant complexation, related fields that have remained largely unconnected from the hydrophobic ion pairing literature. We do not review another related field, nucleotide complexation with cationic lipids to form lipoplexes or solid lipid nanoparticles, but provide references to a number of excellent reviews. At the end of the article we present tables to organize the reported results of hydrophobic ion pairing used for encapsulation. The tables are sorted by both therapeutic and counterion for easy reference and rapid comparison (Fig. 1).

Hydrophobic ion pairing is the process of forming ionic interactions ${ }^{\mathbf{1 0}}$ between a charged hydrophilic molecule with an oppositely-charged counterion. ${ }^{1}$ The counterion contains at least one hydrophobic domain such as an alkyl tail or aromatic ring. The complexation increases hydrophobicity by two main mechanisms: first, the molecule's natural charge is masked, mitigating solubility in polar solvents such as water. Second, the hydrophobic groups on the counterion, typically nonpolar aliphatic tails or aromatic groups, help to coat the original molecule's surface area with hydrophobic domains that exclude water.

For our purposes, the charged hydrophilic is a drug or dye and may be referred to as an 'active pharmaceutical ingredient (API)' or 'therapeutic.' The counterion is referred to in the literature as a 'hydrophobic counterion,' 'ion pair(ing agent) (IP),' or 'salt former.' Due to their amphiphilic chemical nature, 
A. example counterion chemistries

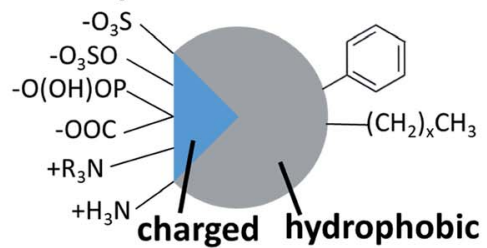

B. stoichiometric ion pairing (1:1 charge ratio)
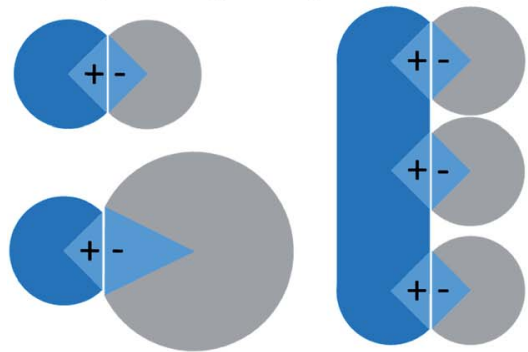

C. nonstoichiometric ion pairing

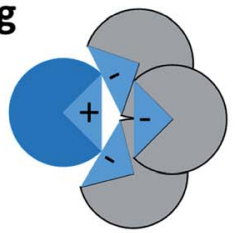
D. ion pairing with inadequate
binding or hydrophobicity
(reversible without salts)

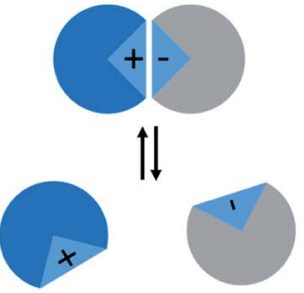

Fig. 1 Hydrophobic ion pairing schematic. (A) Possible charged groups (left) and hydrophobic moieties (right) for a counterion. (B) Stoichiometric ion pairing between a cationic API (blue) and anionic counterion. (C) Non-stoichiometric ion pairing. (D) Reversible ion pairing due to inadequate binding or hydrophobicity.

many hydrophobic counterions used are surfactants, so the term 'surfactant' may be used as well. The act of forming an ionic association between the two species is termed either 'hydrophobic ion pairing' or 'ionic complexation,' and the resulting paired species is a 'hydrophobic complex' or 'HIP complex.' We will discuss later why we do not use the term 'salt.'

Another important piece of terminology is the stoichiometry between the two species. In the HIP literature, there is no standard convention for reporting the ratio of hydrophilic therapeutic to counterion. Molar ratio (reported either as a ratio of $x: y$ or as a fraction), mass ratio, charge ratio, and N/P ratio i.e. ratio of positive to negative charges, usually reported as a fraction, from the lipoplex literature - have all been used. Consider a 1300 Da peptide with five cationic groups that is paired with five molecules of a monovalent counterion of molecular weight 280 Da (Fig. 2A). Reporting ratios as drug:counterion, this complex has a molar ratio of $1: 5$ or 0.2 , a mass ratio of 0.93 , a charge ratio of $1: 1$, and an $\mathrm{N} / \mathrm{P}$ ratio of 1 . Charge and molar ratios are the most intuitive of these, and the $x: y$ ratio nomenclature is more intuitive than fractions.

We recommend that future researchers in the field use charge ratios and report the ratio as 'drug : counterion' rather than as a fraction. Charge ratio is a useful and intuitive parameter in HIP, and should be reported whenever possible. Both molecules' degrees of ionisation may vary with $\mathrm{pH}$; when possible, the charge ratio should be reported at the $\mathrm{pH}$ of the complexation. ${ }^{11}$ When describing the charge ratio of a system where one molecule is zwitterionic, researchers should note whether their reported charge ratio is based on the molecule's net charge or charge of only one type. We recommend the latter, but this is not always possible for large proteins, where only net charge can readily be determined. Consider the example above; if the peptide had one anionic group in addition to five cationic groups, the charge ratio of peptide cations (5) to counterion anions (5) is still 1 : 1 (Fig. 2B). The peptide : counterion charge ratio calculated from the peptide's net charge of $(5-1=) 4$, though, is $4: 5$ or $1: 1.25$, suggesting an excess of counterions when none actually exists. Reporting the molar ratio along with the charge ratio should clarify this point, provided an accurate counting of what charged groups exist on each species is included. In this review, we have converted reported stoichiometries into charge ratios to facilitate comparisons.

\section{Hydrophobic ion pairing}

Hydrophobic ion pairing is an attractive technique for encapsulating water-soluble therapeutics using formulation strategies optimized for water-insoluble drugs. These strategies are desirable because new strategies to encapsulate hydrophilic molecules in nano-scale delivery vehicles remain challenging. ${ }^{12}$ Low drug loadings, poor encapsulation efficiencies, and a lack of scalability continue to prevent many liposome and nanoparticle formulations of biologic therapeutics from reaching the market. ${ }^{12}$ The potential benefits of encapsulation - targeting, protection from enzymatic degradation, improved circulation time, enhanced bioavailability, controlled release, reduced toxicity, and overall improved drug performance - are strong driving motivations to develop scalable, highly-loaded formulations with high encapsulation efficiencies. ${ }^{\mathbf{1 3}, 14}$ This is particularly attractive for biologic (peptide and protein) therapeutics, whose circulation time unprotected in the blood may be as low as minutes. ${ }^{12}$

Nanoparticle formulation strategies for hydrophobic drugs have been developed to address the growing number of new, strongly hydrophobic therapeutics. ${ }^{15,16}$ These techniques - oilin-water emulsions, nanoprecipitation, solid lipid nanoparticles, etc. - are designed to take advantage of a drug's 
A.

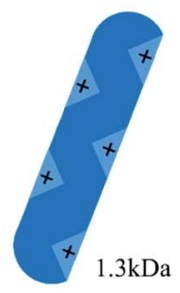

B.

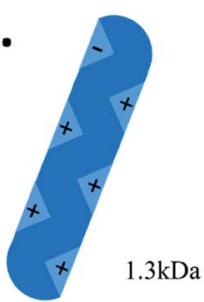

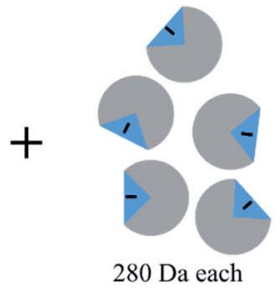

\begin{tabular}{|l|c|}
\hline Mole ratio & $1: 5$ or 0.2 \\
\hline Mass ratio & 0.93 \\
\hline Charge ratio & $1: 1$ \\
\hline N/P ratio & 1 \\
\hline
\end{tabular}

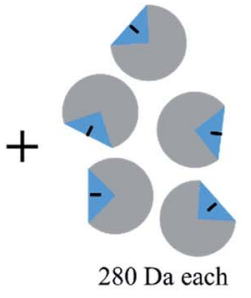

\begin{tabular}{|c|c|}
\hline Mole ratio & $1: 5$ or 0.2 \\
\hline Mass ratio & 0.93 \\
\hline Charge ratio & $\begin{array}{c}\left.1: 1{\left(\mathrm{API}^{+} \text {:surfactant }\right.}^{-}\right) \\
1: 1.25\left(\mathrm{API}^{\text {net }} \text { surfactant }^{-}\right)\end{array}$ \\
\hline
\end{tabular}

Fig. 2 Example schematic of hydrophobic ion pairing between a $1.3 \mathrm{kDa}$ peptide and $280 \mathrm{Da}$ anionic surfactants. When reporting charge ratio, it is helpful to specify if the value given is based on the APl's net charge (typical for proteins) or total number of one kind of charge.

hydrophobicity/lipophilicity. They do not translate easily to the encapsulation of hydrophilic therapeutics. ${ }^{17}$ HIP solves this problem by temporarily modifying the therapeutics to increase their hydrophobicity and allow encapsulation. When the modification is undone, the original hydrophilic therapeutic is regenerated. ${ }^{1}$ We will discuss ways of controlling dissociation to tune release elsewhere in the review. In many reported cases, the de-complexed released therapeutic remains fully active; this has been shown even for large proteins with tertiary structuredependent activity. ${ }^{18}$

Modifying a drug's solubility profile to make it more hydrophobic for encapsulation is also the goal of some prodrug strategies ${ }^{19}$ both techniques temporarily add hydrophobic groups to a hydrophilic molecule. ${ }^{20}$ Unlike prodrug approaches, HIP does not modify any covalent bonds on the original API. This is important from a regulatory standpoint: prodrugs require full FDA approval but the requirements for hydrophobic ion pairs may not be as stringent, depending on the other changes made to the formulation. ${ }^{21}$

\subsection{Thermodynamics}

Mechanisms of solubilisation in aqueous solution. Why are molecules soluble or insoluble in aqueous solutions? Understanding the fundamental mechanisms of solubilization helps understand the principles behind HIP. The solubility or phase behaviour of a species is determined by entropy and enthalpy. Entropy is the state of disorder in a system and is determined by the statistical number of configurations a system can attain. For small molecules, that entropy is determined by the concentration of the solute in the solvent. The entropic contribution to the chemical potential for a dilute solute in an ideal system is $\mu_{\mathrm{i}}^{\text {ideal }}=\mu_{\mathrm{i} 0}+k T \ln x_{\mathrm{i}}$, where $\mu_{\mathrm{i}}$ is the chemical potential ( $\mu_{\mathrm{io}}$ is the chemical potential of the pure species) and $x_{\mathrm{i}}$ is the molar fraction of the solute. It can be seen that entropy always favours dissolution, i.e. increasing the degrees of freedom in the system is favoured.
There is some subtlety with water as the aqueous solvent, since the hydrogen bonding interactions between water molecules adds an entropy contribution to the water solvent itself. ${ }^{22}$ That entropic contribution determines observations such as the Hofmeister series, where the specific salt cations and anions influence solubility. ${ }^{23}$ For this review, we will ignore this effect, since the concept of counter ion binding and precipitation does not require a detailed understanding of water structure.

Water is a unique solvent and is the strongest of the hydrogen bonding fluids. The polarity of the water molecule gives water a high dielectric constant: $\varepsilon=80$. This is in contrast to the dielectric constant of a hydrophobic oil phase (e.g. dodecane), which will have $\varepsilon=2$. The dielectric constant determines the strength of electrostatic interactions between elementary charges. The interaction energy between a positive and negative charge in solution is $F=\frac{k}{\varepsilon} \frac{q_{1} q_{2}}{r^{2}}$. As the dielectric constant increases, therefore, the force holding ions together decreases. Hydrophobic ion pairs stay insoluble in part because they usually include large nonpolar groups that exclude water from fully solvating the ionic-ionic interaction sites. The hydrophobic ion paired precipitate or core of a NC has a low dielectric constant, which magnifies the strength or the electrostatic attractions. This same concept arises in the protein literature, where the interactions between anionic and cationic peptides in the hydrophobic core of a globular protein enhance its stability. However, the same residues on the surface of a protein would enhance its water solubility. It often remains unclear if any water remains associated with the pair in a nanoparticle core; the best data addressing this question comes from studies of ionomers. ${ }^{24-27}$

\subsection{Eligibility for hydrophobic ion pairing and commonly- used counterions}

Eligibility for hydrophobic ion pairing. For a therapeutic molecule to be eligible for HIP, it must contain at least one charged group. Many antibiotics contain amine groups that are 
positively charged at physiological $\mathrm{pH}$ and can be used for this purpose. Anionic carboxylic acid groups are also commonly used as pairing sites in HIP. Molecules with a strong net charge or only one type of charge are the most straightforward to complex, since a single counterion species can be used. Zwitterionic molecules with both anionic and cationic charges present a more complicated challenge; here, shifting the solution $\mathrm{pH}$ to turn off one type of charge can be an effective strategy. This is presented in more detail below. Another consideration for HIP eligibility is the molecule's charge density (charge per molecular weight). Intuitively, adding a single hydrophobic counterion onto a small molecule that has a single charge and molecular weight approximately 300 Da will increase hydrophobicity more than adding the same single counterion to a peptide with one charge and a molecular weight of $3000 \mathrm{Da}$, i.e. the charge per surface area is lower on the larger molecule. Depending on the desired hydrophobicity of the final complex, a given charged molecule may have too low of a charge density, such that adding a hydrophobic counterion will not sufficiently increase hydrophobicity to affect precipitation.

We typically do not use or recommend using the term 'salt' to describe the complexes formed by hydrophobic ion pairing, because 'salts' are commonly understood to refer to crystalline assemblies of stoichiometric amounts of oppositely-charged ions. HIP complexes may be less crystalline than the original drug used, ${ }^{28-30}$ and non-stoichiometric charge ratios are common.

We pause here to briefly address the field of nucleic acid encapsulation and delivery. Nucleic acids - plasmid DNA, linear DNA, siRNA, mRNA, etc. - have been packaged into solid lipid nanoparticles (SLNs) or lipoplexes through ionic complexation between cationic lipids and the nucleic acid's anionic phosphate backbone. This strategy shares a number of similarities with hydrophobic ion pairing, with a few notable exceptions. The most significant is that the regular charge along the phosphate backbone gives nucleic acids a strong, uniform charge density along the molecule. This is different from the small molecule, peptide and protein therapeutics discussed here, which often have less ordered regions of hydrophobicity and hydrophilicity/charge. For the reader familiar with HIP but not SLNs/lipoplexes, we recommend a number of reviews. ${ }^{31-37}$

Common counterions. Because the counterions used for hydrophobic ion pairing should contain at least one charged group and at least one hydrophobic domain, ionic surfactants are common. These may be either anionic or cationic and typically contain either one or two charged groups. Fatty acids or other carboxylic acid-containing surfactants such as oleic acid, stearic acid, or deoxycholic acid, or their sodium salts, have been extensively used. Pamoic acid, which has two carboxylic acid groups, has been effective in cases where fatty acids were not. ${ }^{38}$ Sulfates are also popular, most frequently sodium dodecyl sulfate and sodium docusate. Two-tailed phospholipids such as dimyristoyl phosphatidyl glycerol have been used as well. Anionic polymers such as dextran sulfate have also been investigated, most frequently for complexation with multivalent peptide or protein therapeutics. Though HIP language has been used to describe this kind of polyelectrolyte complexation, the mechanisms differ in important ways. That distinction is the subject of Section 5 of this review (Table 1).

The most common cations in the HIP literature are quaternary amines and alkylamines (see Table 2). Quaternary amines are permanently charged, so complexation is possible over a wider range of $\mathrm{pH}$ values than primary, secondary, or tertiary amines. The permanent charge is usually cytotoxic, and using quatamines adds toxicity to otherwise nontoxic formulations. ${ }^{39}$ A wide variety of quaternary amines is commercially available, with varying lengths and numbers of alkyl tails that lead to an easily tuneable range of hydrophobicities. ${ }^{40}$ Researchers have recently reported efforts to synthesize arginine-based cationic surfactants for HIP, which should be both biodegradable and non-cytotoxic. ${ }^{\mathbf{4 1}}$

\section{Specific considerations by drug molecule class}

Small molecules. Many small molecule drugs have only one ionic group. Depending on the $\mathrm{p} K_{\mathrm{a}}$ of the ionic group and the drug's solubility, HIP is relatively straightforward and can be carried out in water. In a typical 'pre-forming' scenario for hydrophilic small molecules, the drug and counterion are each dissolved in water and mixed to form a precipitate. ${ }^{11,42}$ It is worth noting that small molecules with ionizable groups may be manufactured either as a salt or in the free acid/base form. The free acid/base is usually less soluble in water than the salt, but might not be hydrophobic enough for a desired encapsulation strategy. ${ }^{43}$ Since species must be charged in order to ion pair, salt forms of the drug and hydrophobic counterion may be preferred. When the drug is manufactured in the free acid/base form, conversion to a readily-dissociating salt form (e.g. mesylate, ammonium, or sodium) before HIP may assist complexation. A drawback of this approach is that it increases the solution's overall ionic strength, which can drive decomplexation and drug release from a delivery vehicle by ion exchange. ${ }^{\mathbf{3 8 , 4 4 , 4 5}}$ Researchers should examine the effect of ionic strength on their specific systems to determine if one charge equivalent of soluble counterions such as sodium or ammonium will noticeably affect release.

Some ionic small molecule drugs such as lumefantrine (for structure, see Table 3) are already hydrophobic, so it is not possible to form an aqueous solution as the starting point for HIP. Hydrophobic ion pairing an already-hydrophobic drug can be useful - for example, to decrease drug crystallinity ${ }^{30,46,47}$ - but the complex formation is more challenging. Lumefantrine's tertiary amine has a $\mathrm{p} K_{\mathrm{a}}$ of 8.7 , but the drug's $\log P$ of 9.2 severely limits its ability to dissolve, and the amine to become charged, in water. ${ }^{48}$ Dissolving lumefantrine free base in a nonpolar solvent such as tetrahydrofuran guarantees dissolution, but the extent of the amine's charge is more difficult to control and measure in a non-aqueous environment. As mentioned above, conversion to a salt form before complexation may be useful (Table 4).

Peptides. Many antibiotic peptides such as nisin and colistin (for structure, see Table 3) are cationic and strongly water soluble, with $\log P$ values less than 0 . Basic amino acid residues in the peptide (lysine, histidine, arginine) are positively charged at physiological or acidic $\mathrm{pH}$ and are sites for ion pairing. Some cationic peptide drugs are manufactured as sulfate salts that 
Table 1 Example anionic counterions used in hydrophobic ion pairing

Name Structure

1-Hydroxy-2-naphthoic acid (xinafoic acid)

2-Naphthalene sulfonic acid (NSA)

Brilliant blue FCF

Carboxy methyl polyethylene glycol (CM-PEG)

Cholesteryl hemisuccinate

Cholic acid (sodium cholate)

Decanoic acid (sodium decanoate/sodium caprate)

Dimyristoyl phosphatidyl glycerol (DMPG)

Dioleoyl phosphatidic acid (DOPA)

Docosahexaenoic acid

Hexadecylphosphate

Linoleic acid

$N, N$-Dipalmitoyl-L-lysine Structure<smiles>O=C(O)c1ccc2ccccc2c1O</smiles>

188.2

3.02

2.6 AZD2811 (ref. 38 and 89)<smiles>O=S(=O)(O)c1ccc2ccccc2c1</smiles>

208.2

$$
-1.8
$$

2.14 Atazanavir ${ }^{28}$

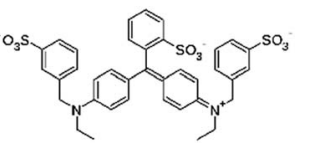

792.8

5.83<smiles>CC(C)(C)COCC(=O)O</smiles>

PEG length not given

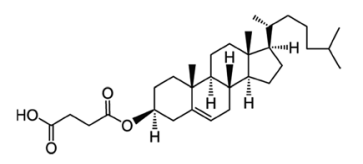

486.7

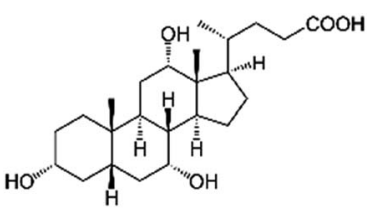

408.6<smiles>CCCCCCCCCC(=O)O</smiles>

194.3

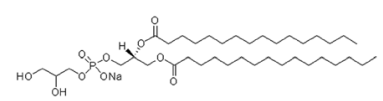

666.9

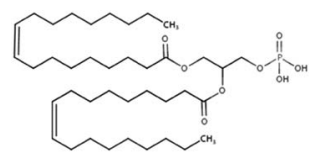

701<smiles>C/C=C\C/C=C\CC/C=C\CCCC(=O)C(C)C</smiles>

328.5

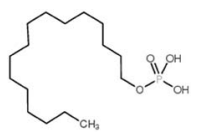

320.4

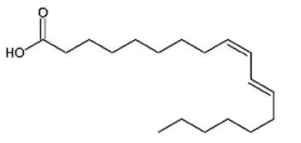

280.5 and

6.58

4.98

5.8

-1.45 Atenolol $^{155}$

Bovine serum albumin ${ }^{56}$

Lysozyme $^{56}$

r-met-HuGdNF ${ }^{56}$

Colistin $^{156}$

Doxorubicin $^{112}$

8.5

AZD2811 (ref. 38 and 89) Bovine serum albumin ${ }^{56}$ Lysozyme $^{56}$ 2.02 r-met-HuGdNF ${ }^{56}$ Insulin $^{157}$

4.9

4.09 Octreotide ${ }^{9,96}$

1.89

Insulin $^{42}$

9.2 Salmon calcitonin ${ }^{85}$

Gefitinib $^{30}$

$1.3 \quad 13.2$

4.89

6.75 Doxorubicin $^{70}$

Doxorubicin $^{158}$

6.38 Tobramycin $^{160}$

Colistin $^{156}$
Doxorubicin $^{71}$

Thymopentin $^{159}$ 
Table 1 (Contd.)

Name Structur

Oleic acid (sodium oleate also used)

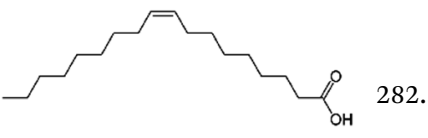

Pamoic acid (disodium pamoate also used)

Sodium acetate

Sodium cholesteryl sulfate

Sodium decanesulfonate (SDES)

Sodium deoxycholate

Sodium docusate (AOT, sodium dioctyl sulfosuccinic acid, sodium bis-2- ethylhexyl-sulfosuccinate)<smiles>O=C(O)c1cc2ccccc2c(Cc2c(O)c(C(=O)O)cc3ccccc23)c1O</smiles>

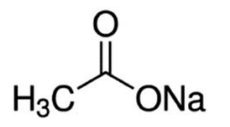

82

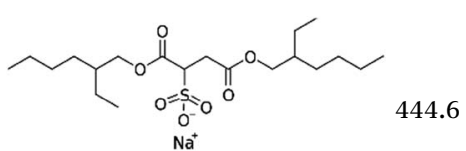

AZD2811 (ref. 38 and 89) Berberine $^{161}$

Desmopressin ${ }^{77,86}$

Dorzolamide ${ }^{81}$

Doxorubicin ${ }^{106,121}$

Insulin ${ }^{86,102,162}$

Leuprolide s6,94,101 $^{8}$

6.78 Lumefantrine ${ }^{48}$

Lycobetaine ${ }^{163}$

Lysozyme $e^{57,58}$

Octreotide $^{96}$

OZ439 (ref. 43 and 154)

Polymyxin $\mathrm{B}^{78}$

Salmon calcitonin ${ }^{85}$

Vincristine ${ }^{164}$

AZD2811 (ref. 38)

$388.4 \quad 2.68$

albumin $^{165}$

Cinnarizine $^{47}$

6.17 Clozapine $^{47}$

Donepezil $^{166}$

Insulin ${ }^{165}$

Leuprolide $\mathrm{e}^{165}$

Polymyxin $\mathrm{B}^{78}$

Doxorubicin $^{61}$

4.7

Propanolol $^{61}$

-0.2 Quinidine sulfate ${ }^{61}$

Verapamil $^{61}$

466.3

3.13

4.2 Colistin $^{156}$

3.75 Doxorubicin ${ }^{116}$

AZD2811 (ref. 38)

Bovine serum

albumin $^{165}$

Ciprofloxacin $^{88}$

Insulin $^{80,93,165}$

$4.65 \quad 3.8$ Lanreotide $^{98}$

Leuprolide $^{165}$

Mitoxantrone $\mathrm{diHCl}^{79}$

Octreotide $^{9,96}$

Papain $^{97}$

Salmon calcitonin ${ }^{85}$

$\alpha$-Chymotrypsin ${ }^{167}$

Atazanavir $^{28}$

AZD2811 (ref. 38 and 89)

Bevacizumab $^{168}$

Bovine serum

albumin $^{56,169}$

Cisplatin $^{83}$

Concanavalin $\mathrm{A}^{167}$

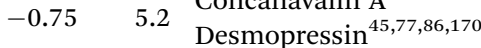

Doxorubicin $^{116}$

Gentamycin $^{82,117-119,171}$

Irinotecan $^{29}$

Lanreotide $^{98}$

Leuprolide $^{45,84,86,110,172}$

Lysozyme $^{56}$

Minocycline ${ }^{173}$ 
Table 1 (Contd.)

Nam Structure MW, Da $\mathrm{p} K_{\mathrm{a}}$

$\log P \quad$ Used to pair with

Mtb8.4 (ref. 174)

Naloxone ${ }^{117}$

Naltrexone $\mathrm{e}^{117}$

Octreotide $^{9}$

r-met-HuGdNF ${ }^{56}$

Tobramycin ${ }^{175}$

Trypsin $^{167}$

Vancomycin ${ }^{176}$

Sodium dodecyl benzenesulfonate (SDBS)

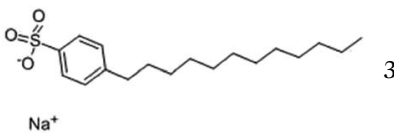

348.5

$-1.7 \quad 3.73$ Polymyxin $\mathrm{B}^{78}$

$\mathrm{Na}^{+}$

Sodium dodecyl sulfate (sodium lauryl sulfate)

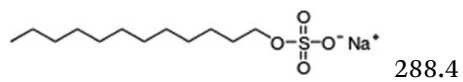

$-1.5$

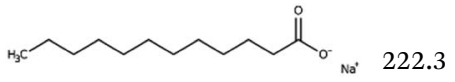

4.95

Bovine serum albumin ${ }^{56}$ Desmopressin $^{77,86}$

Dorzolamide $^{81}$ IGG-Fab fragment ${ }^{90}$ Insulin $^{49,58,84,86,111,177,178}$

Sodium laurate (sodium dodecanoate)

$$
\mathrm{H}_{3} \mathrm{C}
$$

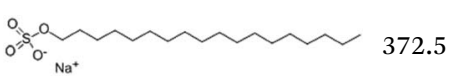<smiles>CCCCCCCCCCCCCCCCCC(=O)[O-]</smiles>

4.7 Irinotecan $^{29}$ Sodium n-octadecyl sulfate (sodium stearyl sulfate)

Sodium stearate (stearic acid also used)

Sodium stearoyl glutamate (SSG)<smiles>CCCCCCCCCCCCCCCCC(=O)NC(CCC(=O)O)O[C@H](O)C(=O)O</smiles>

Sodium taurodeoxycholate (STDC)

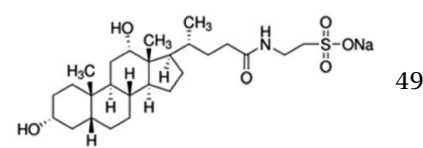

499.7

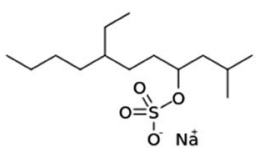

316.4

$-0.94$

4.5

\section{Bovine serum}

6.3 albumin $^{165}$

Insulin $^{165}$

Leuprolide $^{165}$

Doxorubicin $^{72,95,116}$

Idarubicin $^{95}$

Melittin $^{109}$

Octreotide ${ }^{92,96}$

Polymyxin $\mathrm{B}^{78}$

r-met-HuGdNF ${ }^{56}$

5.3 albumin $^{165}$

Leuprolide $^{165}$

Desmopressin $^{77}$

6.8 Lanreotide $^{98}$

Desmopressin ${ }^{77}$

Doxorubicin $^{61}$

Propanolol $^{61}$

8.23 Quinidine sulfate ${ }^{61}$

Verapamil $^{61}$

Sodium tetradecyl sulfate

Nà

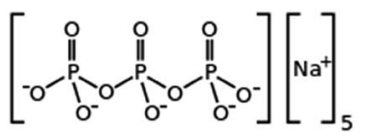

367.9

0.89

Sodium tripolyphosphate 
Table 1 (Contd.)

Name
Taurocholic acid (sodium taurocholate also used)
Vitamin E ( $\alpha$-tocopherol) succinate

dissociate readily in water and do not have the same solubility and ionization challenges as hydrophobic small molecules. For peptides with only cationic charges such as polymyxin B, aqueous complexation with anionic surfactants is straightforward. Zwitterionic peptides are more challenging, however. If a peptide contains both cationic and anionic groups, it is possible that complexing only the cationic sites and leaving anionic sites charged and exposed (or vice versa) will impart sufficient hydrophobicity for the desired application. This is especially true when one kind of charged site significantly outnumbers the other, as in the case of a COOH-terminated peptide with five cationic sites. Complexing five out of the six charged sites with hydrophobic counterions may reduce water solubility enough to enable encapsulation.

When there are approximately the same number of cationic and anionic sites on a zwitterionic peptide, though, complexing only one charge may not be sufficient. It is preferable to use only one counterion species to complex a molecule, rather than adding both anionic and cationic hydrophobic counterions (which will invariably pair with each other and precipitate, complicating stoichiometry and adding difficult-to-separate insoluble salts to the system) in an attempt to complex every charged site. In this case, shifting the $\mathrm{pH}$ to turn off one type of charge is a valid approach. Consider insulin, a $5.8 \mathrm{kDa}$ peptide with 51 residues, 6 of which are cationic and 6 anionic. Insulin has no net charge at its isoelectric point at $\mathrm{pH}$ 5.3. Researchers have reported shifting the solution $\mathrm{pH}$ either up or down from 5.3 to deprotonate insulin's basic residues or protonate its acidic residues, respectively. ${ }^{42,49-53}$ With only one type of charge, the peptide can then be hydrophobically ion paired (Fig. 3).

Researchers should consider several factors when using a $\mathrm{pH}$ shifting strategy. First, peptides are subject to degradation under basic conditions, so shifting the $\mathrm{pH}$ to strongly acidic is likely preferable. ${ }^{54}$ Second, the complexing counterions are subject to protonation or deprotonation under extreme $\mathrm{pH}$ conditions as well. Insulin has only cationic charges at $\mathrm{pH} 1.5$, but an anionic fatty acid counterion such as oleic acid $\left(\mathrm{p} K_{\mathrm{a}} \sim 5\right)$ will be protonated under those conditions too. A much more acidic counterion such as sodium dodecyl sulfate $\left(\mathrm{p} K_{\mathrm{a}}-1.5\right)$ or sodium docusate $\left(\mathrm{p} K_{\mathrm{a}}-0.75\right)$ must be used. These sulfate surfactants are less biocompatible than fatty acids, in part because of this difference in $\mathrm{p} K_{\mathrm{a}}$. The same considerations apply when shifting the $\mathrm{pH}$ to basic. Quaternary amines may be the only groups to reliably retain their cationic charge at a high $\mathrm{pH}$, but using these cytotoxic surfactants to complex an anionic peptide presents its own challenges.

Proteins. Protein therapeutics are commonly zwitterionic, and all the considerations of net charge, ratio of basic to acidic residues, pI, and pH shifting that apply to zwitterionic peptides also apply to proteins. An additional complication when complexing proteins is their sensitivity to denaturation. Some surfactants such as sodium dodecyl sulfate disrupt tertiary structure and cause proteins to denature. ${ }^{55}$ Using 'gentler' surfactants such as fatty acids may cause less degradation, but might also prevent the $\mathrm{pH}$ shifting approaches discussed above.

A popular model protein for hydrophobic ion pairing and encapsulation is lysozyme, which is cationic at physiological pH. ${ }^{18,56-59}$ Lysozyme's enzymatic activity can be easily measured via a cell lysis assay; therefore, testing whether or not the protein was denatured during complexation, encapsulation, and release is straightforward. Devrim et al. found that even when using sodium dodecyl sulfate as an ion pairing agent, released lysozyme retained over $80 \%$ of its enzymatic activity. ${ }^{\mathbf{1 8}}$ Yoo et al. reported that the enzyme was more stable in DMSO when ion paired using SDS or oleate, and postulated that HIP complexation could help stabilize a protein's tertiary structure. ${ }^{57}$ Notably, lysozyme tends to refold into its native active form, so not all techniques that claim to 'retain' the protein's activity will do so for all enzymes.

\subsection{Key parameters for hydrophobic ion pairing}

The following section is intended to guide the reader in choosing an effective hydrophobic counterion for a given encapsulation and/or delivery system. It is important to note that the goals for a given delivery system - e.g. drug chemistry, drug loading, encapsulation technique, biological target, release profile, etc. - are the most important factors when choosing a suitable counterion. This section will overview how parameters such as drug : counterion charge ratio and counterion chemistry affect those goals.

Counterion chemistry: hydrophobicity. The $\log P$, the logarithm of the octanol-water partition coefficient, is a typical measure of hydrophobicity that is convenient for HIP. For 
Table 2 Example cationic counterions used in hydrophobic ion pairing

Name

Arginine-hexadecanoyl ester (AHE)

Arginine-nonyl ester (ANE)

Benethamine( $N$-benzyl-2-phenylethanamine)

Chitosan

Dodecylamine (laurylamine)

Hexadecyl trimethylammonium(cetrimonium) bromide (CTAB)

Maprotiline

$N^{\alpha}$-Deoxycholyl-L-lysyl-methylester

$N, N^{\prime}$-Dibenzyl ethylenediamine(benzathine)

$N, N$-Dimethyl dodecylamine (DDA)

$N, N$-Dimethyl hexylamine

$N, N$-Dimethyl octadecylamine(dimethyl stearamine)

Stearylamine(octadecylamine)

Tetrabutyl ammonium bromide (TBAB)

Tetraheptyl ammonium bromide (THA)

Tetrahexyl ammonium bromide
Structure

Mol. wt. $\mathrm{p} K_{\mathrm{a}} \log P \quad$ Paired with

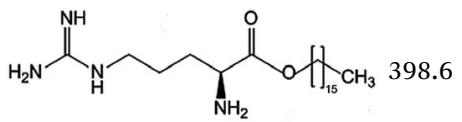

Daptomycin ${ }^{41}$

0.19 Heparin $^{41}$<smiles>CC(C)OC(=O)[C@H](N)CCCNC(=N)N</smiles>

Daptomycin $^{41}$ -0.06 Heparin $^{41}$

211.3

3.6 Retinoic acid a5,108,115 $^{6}$<smiles>c1ccc(CCNCc2ccccc2)cc1</smiles>

Varies

Insulin $^{53}$

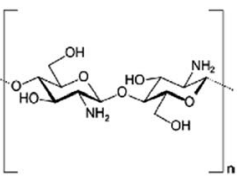

$\underbrace{\mathrm{CH}_{3}}_{\mathrm{H}_{2} \mathrm{~N}} 185.3 \quad 10.65 .2 \operatorname{Retinoic}^{18 \operatorname{acid}^{46,107,114}}$

Ovalbumin ${ }^{39}$

Pemetrexed $^{103}$

Poly(I:C $)^{39}$<smiles>CNCCCC12CC(c3ccccc31)c1ccccc12</smiles>

277.4

10.5 5.1 Retinoic acid $^{115}$

3.8 Pemetrexed ${ }^{4}$<smiles>CCCCCCC(NC(=O)CCC1CCC2C3CC[C@@H]4CC(O)CCC4(C3)C1CC(C)C2C)C(C)=O</smiles>

240.3

$2.86 \alpha$-Lipoic acid ${ }^{47}$<smiles>c1ccc(CNCCNCc2ccccc2)cc1</smiles>

213.4

9.975 .91 Am80 (ref. 40)<smiles>CCCCCCN(C)C</smiles>

$129.2 \quad 10.42 .72 \quad$ Am80 (ref. 40)

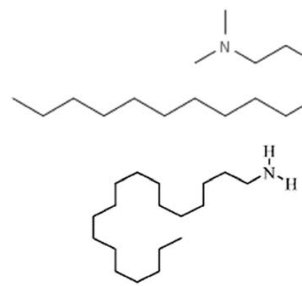

297.6

8.8 Am80 (ref. 40)

269.5

10.7 7.7 Retinoic acid a6,65,107,114 $^{46}$

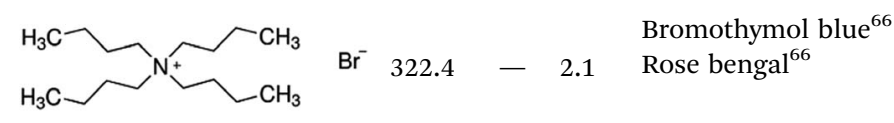

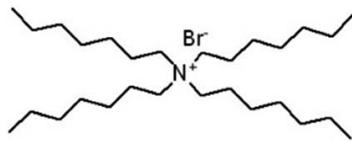

$490.7-8.16$ Isoniazid

methanesulfonate ${ }^{179}$

Bromothymol blue ${ }^{66}$

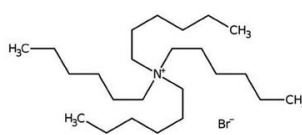
Rose bengal $^{66}$ 
Table 2 (Contd.)

Tetrapentyl ammonium bromide (TPA)

a given charged head group, the longer or more saturated an alkyl tail, or the more alkyl tails, the higher the $\log P$. Stearic acid (lipid number $18: 0$ ), for example, has a higher $\log P$ than both capric acid (lipid number 10:0) and oleic acid (lipid number $18: 1$ ). Quaternary amines also follow this trend, though their alkyl tails are fully saturated. Dimethyl dihexadecyl ammonium bromide (two methyl tails and two $\mathrm{C}_{16}$ tails) is more hydrophobic than CTAB (three methyl tails and one $\mathrm{C}_{16}$ tail), and tetraheptyl ammonium bromide (four $\mathrm{C}_{7}$ tails) is more hydrophobic than tetrabutyl ammonium bromide (four $\mathrm{C}_{4}$ tails). Note that $\log P$ values for a free acid/base or an ionized surfactant may be different when reported from measurements or calculations. In general, the higher the $\log P$ of the counterion used, the higher the $\log P$ of the resulting complex. ${ }^{40,60}$

The most hydrophobic counterion is not always the best to use. Increasing alkyl tail length or number of tails increases molecular weight, meaning the final complex will have a lower mass fraction of drug. This drives down drug loading in a delivery vehicle, all else (charge ratio, encapsulation efficiency, etc.) being equal. Availability and cost are another factor, since not all fatty acids or quaternary amines are commercially available at high purity and low cost. Solubility limitations are discussed in the following paragraph. Finally, comparing $\log P$ values among fatty acids is straightforward, but it is difficult a priori to compare the effect of a fatty acid $v s$. a bile acid or other carboxylic acid surfactant (e.g. oleic acid vs. pamoic acid, which is divalent) on complexation.

Extremely hydrophobic counterions, particularly those with protonated (free acid) carboxylic head groups, are difficult to dissolve in water for ion pairing. For the pairing to be effective, care should be taken to ensure that both species are dissolved and ionized prior to complexation. We recommend using a counterion's most water-soluble salt form, usually a sodium salt for anions and a bromide salt for cations. ${ }^{61}$ For example, oleic acid is sparingly soluble in water, but sodium oleate is water-soluble up to $10 \mathrm{wt} \% .^{62,63}$

When choosing among different counterions with various $\log P$ values, it is important to keep in mind why HIP is needed. This will vary by the encapsulation technique used. For example, when using nanoprecipitation, the primary goal of complexation is to decrease water solubility. When using an emulsion or SLN approach, however, the main goal is to increase lipophilicity. These distinctions will be discussed in further detail in Section 3, which focuses on encapsulation strategies, but we will give a brief example here. Consider vancomycin, a 1450 Da peptide with a single ionisable primary amine. We have found that vancomycin cannot be made to precipitate in Flash NanoPrecipitation, even using HIP, due to its low charge density. Kalhapure et al., however, improved vancomycin encapsulation efficiency from $16.8 \%$ to and $70.7 \%$ by preforming a vancomycin : linoleic acid complex prior to formulation by hot homogenization and ultrasonication using the solid lipid Compritol 888 ATO and additional surfactants. ${ }^{64}$ It is likely that vancomycin's increased lipophilicity, rather than improved hydrophobicity, led to this result. Adding oleate's 18carbon tail to vancomycin likely improved the API's ability to interact with and remain associated with Compritol 888's alkyl tail.

A counterion's $\log P$ value is therefore not the only factor to consider when considering hydrophobicity. ${ }^{56}$ It is important to remember that in addition to excluding water, hydrophobic domains on a counterion can interact hydrophobically and sterically with (1) one another, (2) hydrophobic domains on the complexed drug, and (3) the delivery vehicle's polymers, lipids, or surfactants. ${ }^{65,66}$ Hydrophobic interactions may make a counterion with aromatic groups more suitable for use than one with an aliphatic tail, for example, or give rise to favourable cooperativity between a drug and counterion with an unsaturated aliphatic tail, even though one with a saturated tail may have a higher $\log P$. These interactions remain an active area of research.

Counterion chemistry: $\mathrm{p} \boldsymbol{K}_{\mathrm{a}}$ and $\mathrm{pH}$. Counterions must be charged to ionically complex. Sulfate and sulfonate anions and quaternary amine cations are essentially always charged in aqueous environments, but the degree of ionization for carboxylic acids and primary, secondary, and tertiary amines varies with $\mathrm{pH}$. Therefore $\mathrm{pH}$ and $\mathrm{p} K_{\mathrm{a}}$ (of the drug molecule and the counterion) are both important to consider during HIP. Operating at a $\mathrm{pH}$ near one species' $\mathrm{p} K_{\mathrm{a}}$ value is not advised, because charge ratios are difficult to predict and control 
Table 3 Examples of hydrophobic ion pairing, sorted by therapeutic

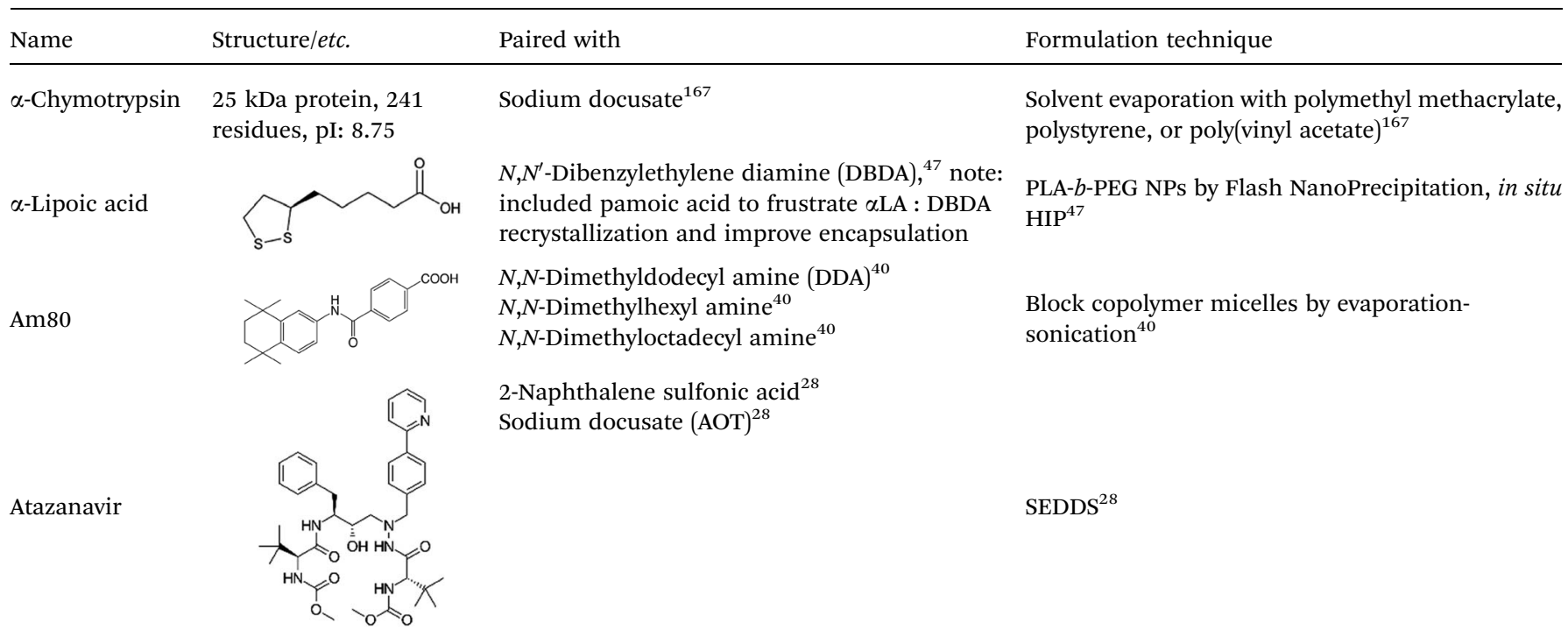

Atenolol

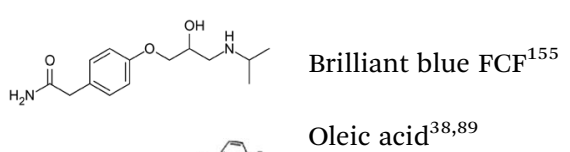

AZD2811

Bevacizumab Bovine serum albumin (BSA)

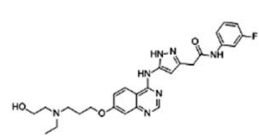

Oleic acid 38,89 $^{30}$

1-Hydroxy-2-naphthoic acid ${ }^{38,89}$

Cholic acid ${ }^{38,89}$

Sodium deoxycholate ${ }^{38}$

Docusate sodium ${ }^{38,89}$

Pamoic acid $^{38}$

Oleic $\operatorname{acid}^{161}$

Docusate sodium ${ }^{168}$

$149 \mathrm{kDa}$ antibody

$66.5 \mathrm{kDa}$ protein, 583

residues, pI: 4.7

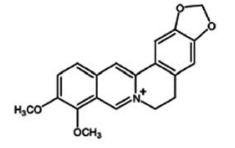

Cholic acid $^{56}$

CM-PEG ${ }^{56}$
Bromothymol

blue

Chlorhexidine

Cinnarizine
Sodium dodecyl sulfate ${ }^{56}$

Taurocholic acid ${ }^{56}$

Sodium docusate ${ }^{56}$

Dextran sulfate ${ }^{91}$

Sodium deoxycholate ${ }^{165}$

Sodium laurate ${ }^{165}$

Sodium stearoyl glutamate ${ }^{165}$

Pamoic acid disodium ${ }^{165}$

Tetrabutylammonium bromide ${ }^{66}$

Tetrahexylammonium bromide ${ }^{66}$

Tetraoctylammonium bromide ${ }^{66}$
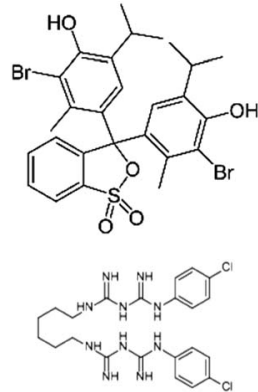

$\operatorname{Losartan}^{152}$

Pamoic acid, ${ }^{47}$ note: Also unsuccessfully tried camphor-10 sulfonic acid (micellized), cinnami acid, palmitic acid, and oleic acid
PLGA NPs by nanoprecipitation ${ }^{155}$

Oil in water $(\mathrm{o} / \mathrm{w})$ nanoemulsification solvent extraction to form PLA-PEG NPs using in situ $\mathrm{HIP}^{38,89}$

Liquid crystalline nanoparticulates by a hydrotrope method ${ }^{161}$

Lipid coacervation ${ }^{168}$

Double emulsion ${ }^{56}$

Single emulsion ${ }^{56}$

Double emulsion ${ }^{56}$

Single emulsion ${ }^{56}$

SEDDS $^{169}$

Solid in oil in water $(\mathrm{S} / \mathrm{O} / \mathrm{W})$ to form PLGA NPs ${ }^{91}$ SEDDS $^{165}$

Encapsulated into polystyrene microparticles using compressed carbon dioxide ${ }^{66}$

Nanoprecipitation $^{152}$

PLA- $b$-PEG NPs by Flash NanoPrecipitation, in situ $\mathrm{HIP}^{47}$ 
Table 3 (Contd.)

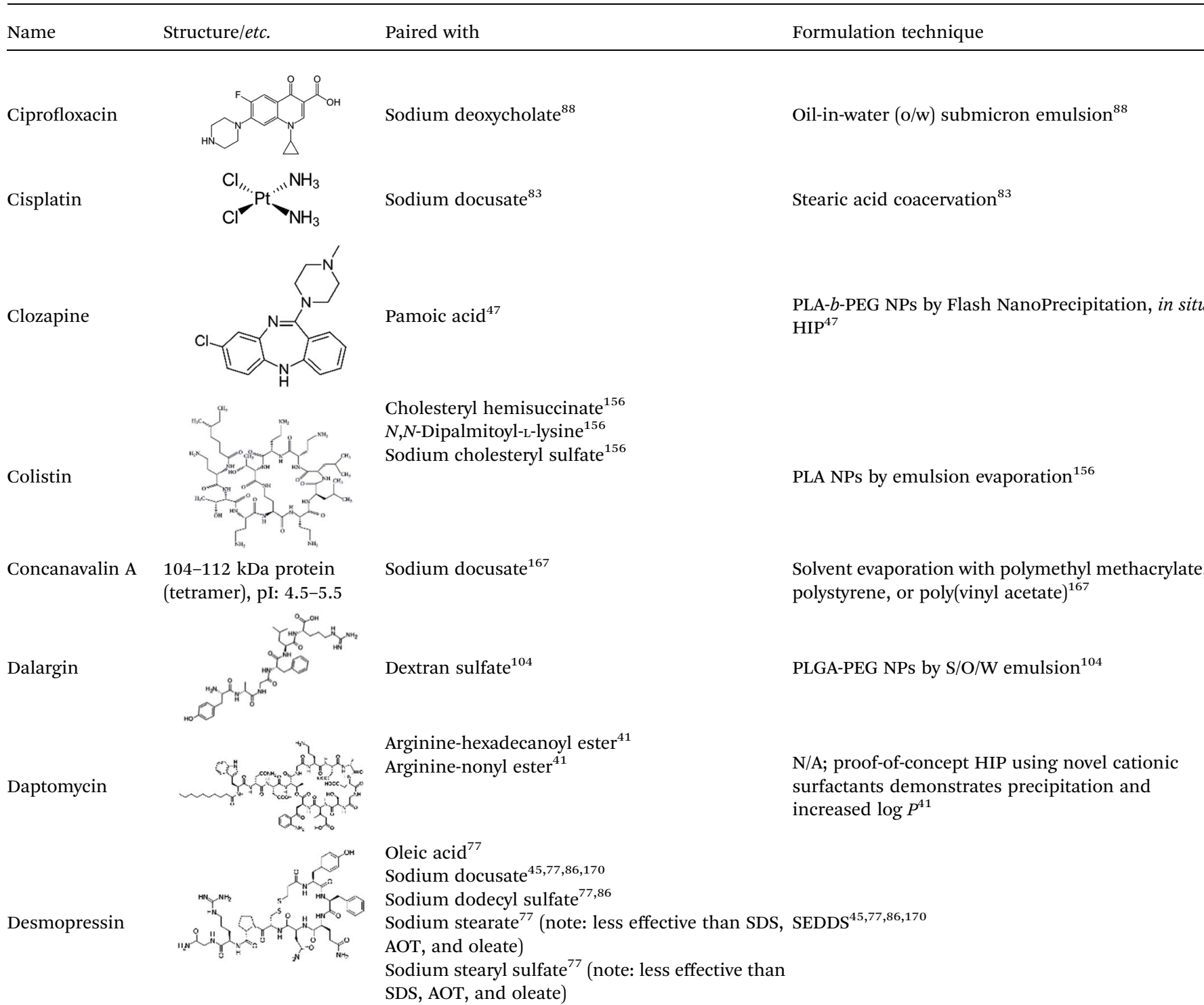

Dexamethasone valine valine prodrug

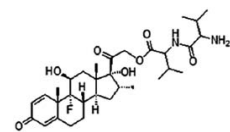

Dextran sulfate ${ }^{120}$

PLGA NPs by $\mathrm{S} / \mathrm{O} / \mathrm{W}$ emulsion ${ }^{120}$

Donepezil

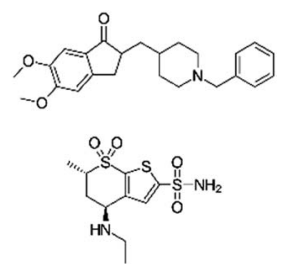

Pamoic acid $^{166}$

High pressure homogenization with $\mathrm{D}-\alpha-$ tocopherol polyethylene glycol 1000 succinate $^{166}$

Oleic acid ${ }^{81}$

Sodium dodecyl sulfate ${ }^{81}$

PLGA NPs or PEG3-PSA microparticles by S/O/W emulsion $^{81}$

Microemulsion by stearic acid coacervation ${ }^{61}$ Thin film dispersion ${ }^{112}$

Alginic acid ${ }^{61}$<smiles>COc1cccc2c1C(=O)c1c(O)c3c(c(O)c1C2=O)C[C@H](C(=O)O)C[C@H]3O</smiles>

Cholesteryl hemisuccinate ${ }^{112}$

Dextran sulfate ${ }^{61,95}$

Microemulsion by stearic acid coacervation ${ }^{61}$ Warm wax microemulsion solvent evaporation ${ }^{95}$ PLA- $b$-PEG NPs by nanoprecipitation ${ }^{71}$

Dioleoyl phosphatidic acid (DOPA) ${ }^{71}$

Docosahexaenoic acid 70

Hexadecylphosphate ${ }^{158}$

SLNs by hot melt ultrasound emulsification ${ }^{70}$

SLNs by warm oil-in-water microemulsion with stearic acid and taurocholate sodium ${ }^{158}$

Hyaluronic acid ${ }^{153}$ suspended HIP complex and homogenization ${ }^{153}$ 
Table 3 (Contd.)

\begin{tabular}{|c|c|c|}
\hline Name & Structure/etc. & Paired with \\
\hline & & Oleic acid $^{106,121}$ \\
\hline & & $\begin{array}{l}\text { Sodium acetate }^{61} \\
\text { Sodium alginate }^{61} \\
\text { Sodium decanesulfonate }^{116} \\
\text { Sodium docusate } \\
\text { Sodium stearate } \\
\text { Sodium taurodeoxycholate }^{72,95,116}\end{array}$ \\
\hline
\end{tabular}

Gefitinib<smiles>Oc1cc2ncnc(Nc3ccc(F)c(Cl)c3)c2cc1O</smiles>

Sodium tetradecyl sulfate ${ }^{95}$

Vitamin E succinate ${ }^{105}$

Dioleoyl phosphatidic acid (DOPA) ${ }^{30}$

Gentamicin

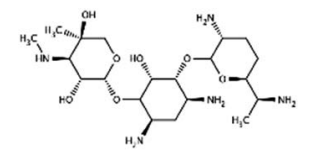

Sodium docusate $\mathrm{e}^{82,117-119,171}$

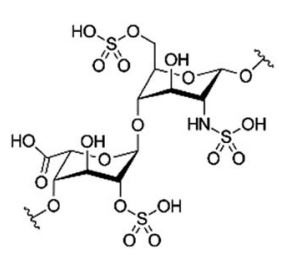

Arginine-hexadecanoyl ester ${ }^{41}$

Heparin

Arginine-nonyl ester ${ }^{41}$

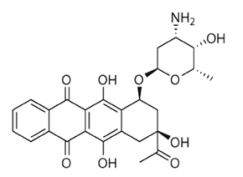

Dextran sulfate ${ }^{95}$

Sodium taurodeoxycholate ${ }^{95}$

Sodium tetradecyl sulfate ${ }^{95}$

Idarubicin

$\mathrm{HCl}$

IGG-Fab fragment $48 \mathrm{kDa}$ protein

Insulin

$5.8 \mathrm{kDa}$ peptide, 51 residues ( 6 cationic and 6 anionic), pI: 5.3

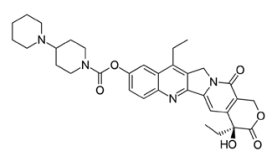

Sodium docusate ${ }^{29}$

Sodium dodecyl sulfate ${ }^{29}$

Sodium tripolyphosphate ${ }^{29}$

Tetraheptylammonium bromide ${ }^{179}$
Formulation technique

$70{ }^{\circ} \mathrm{C}$ high-pressure homogenization ${ }^{121}$

High-pressure film homogenization ${ }^{106}$

Microemulsion by stearic acid coacervation ${ }^{61}$

Microemulsion by stearic acid coacervation ${ }^{61}$

Microemulsion by stearic acid coacervation ${ }^{116}$

Microemulsion by stearic acid coacervation ${ }^{116}$

Microemulsion by stearic acid coacervation ${ }^{61}$

Warm wax microemulsion solvent evaporation ${ }^{95}$

Microemulsion by stearic acid coacervation ${ }^{116}$

Microemulsion by shear and ultrasonic

homogenization after drying from molten stearyl alcohol $^{72}$

Warm wax microemulsion solvent evaporation ${ }^{95}$ SLNs by hot melt ultrasound emulsification ${ }^{105}$

Nanoprecipitation with doxorubicin-conjugated PLA- $b$-PEG NPs ${ }^{30}$

PLA microparticles by precipitation with compressed antisolvent $t^{117,171}$

Microparticles by PCA using stabilizer poly(methyl vinyl ether-co-maleic anhydride) $)^{118}$

PCA with no stabilizer ${ }^{82,119}$

PLGA NPs by emulsion solvent evaporation ${ }^{82,119}$

N/A; proof-of-concept HIP using novel cationic surfactants demonstrates precipitation and increased $\log P^{41}$

Warm wax microemulsion solvent evaporation ${ }^{95}$

Modified nanoprecipitation ${ }^{90}$

S/O/W PLGA NPs ${ }^{90}$

Reverse micelle-double emulsion using palmitic and stearic acid ${ }^{157}$

Homogenization and stabilization with $\mathrm{SDS}^{53}$ SNEDDS $^{42}$

$\mathrm{S} / \mathrm{O} / \mathrm{W}$ emulsion ${ }^{58}$

PLGA NPs by emulsion solvent diffusion ${ }^{102,162}$ SEDDS $^{165}$

PLGA NPs by emulsion solvent diffusion ${ }^{93}$

$\mathrm{S} / \mathrm{O} / \mathrm{W}$ emulsion ${ }^{80}$

SEDDS $^{165}$

SEDDS $^{86}$

Stearic acid coacervation ${ }^{84}$

Stearic acid coacervation ${ }^{84}$

PLGA NPs by emulsion solvent diffusion ${ }^{111,177,178}$

Electrospray with stearic or pamoic acid ${ }^{49}$

PEG- $b$-PLGA NPs via water/oil/water double emulsion; in situ $\mathrm{HIP}^{29}$

Precipitation with compressed antisolvent (PCA $)^{179}$ 
Table 3 (Contd.)

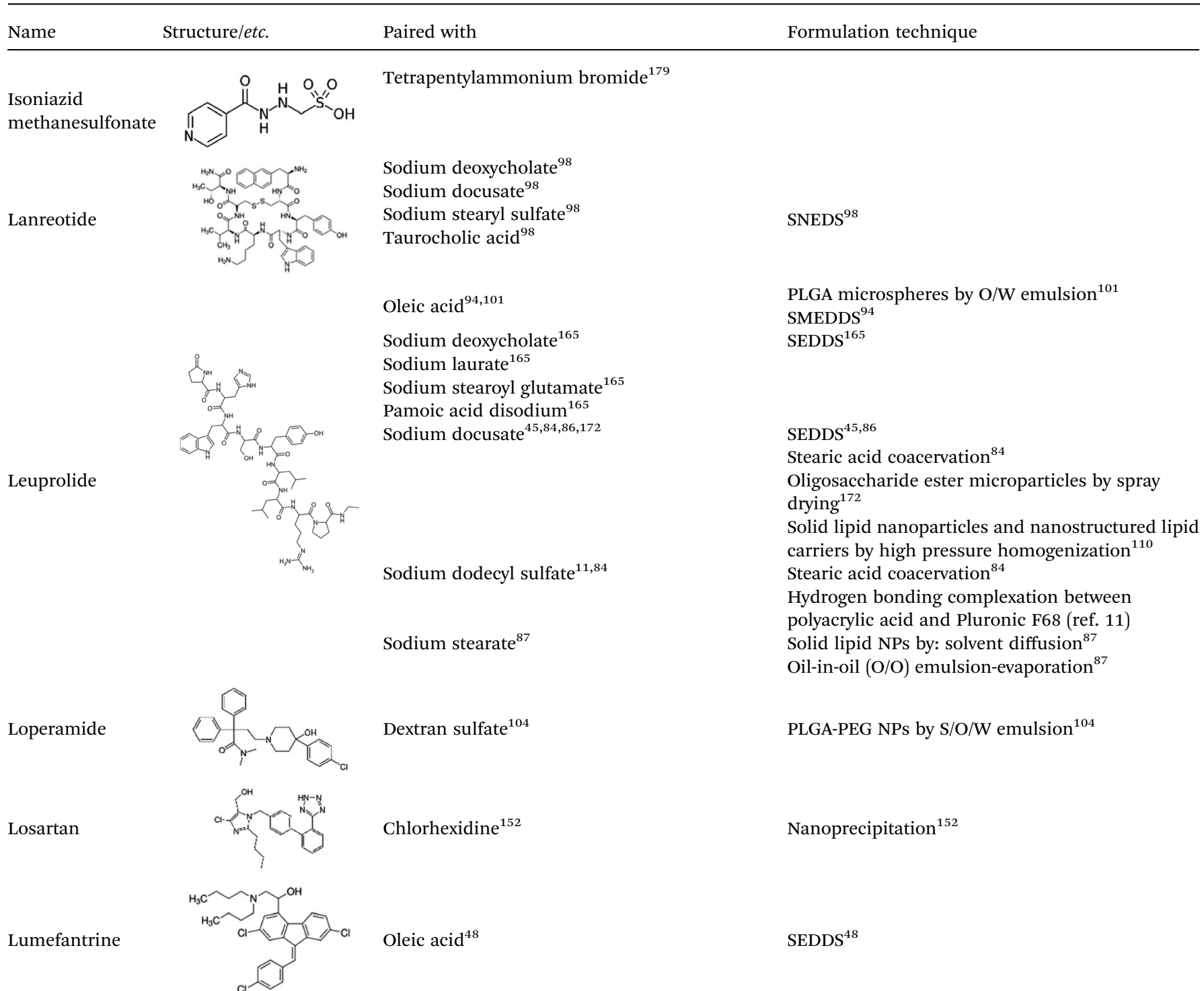

Lycobetaine

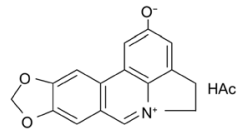

Lysozyme residues, pI: 11.35

Oleic acid ${ }^{163}$

Cholic acid ${ }^{56}$

CM-PEG ${ }^{56}$

Dextran sulfate ${ }^{59}$

Oleic acid a $^{5,58}$

Sodium docusate $\mathrm{e}^{56}$

Sodium dodecyl sulfate ${ }^{18,57}$

Taurocholic acid ${ }^{56}$

Melittin

Minocycline

$2.8 \mathrm{kDa}$ peptide, 26 residues, pI: 12.01

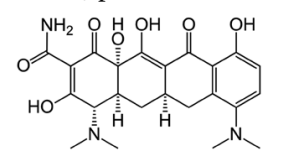

Sodium dodecyl sulfate $e^{109}$

Sodium docusate $\mathrm{e}^{173}$
Emulsion by lipid film hydration high-pressure homogenization $^{163}$

Double emulsion ${ }^{56}$

Single emulsion ${ }^{56}$

Double emulsion ${ }^{56}$

Single emulsion ${ }^{56}$

Emulsion solvent diffusion ${ }^{59}$

PLGA NPs by emulsion diffusion ${ }^{57}$

$\mathrm{S} / \mathrm{O} / \mathrm{W}$ emulsion ${ }^{58}$

Double emulsion ${ }^{56}$

Single emulsion ${ }^{56}$

PLGA NPs by emulsion diffusion ${ }^{57}$

S/O/W emulsion: Polymer/lipid NPs ${ }^{18}$

Double emulsion ${ }^{56}$

Single emulsion ${ }^{56}$

PLGA nanoparticles by emulsion solvent diffusion ${ }^{109}$

PLGA NPs by emulsion-solvent-diffusion ${ }^{173}$ 
Table 3 (Contd.)

\begin{tabular}{|c|c|c|}
\hline Name & Structure/etc. & Paired with \\
\hline
\end{tabular}

Mitoxantrone dihydrochloride

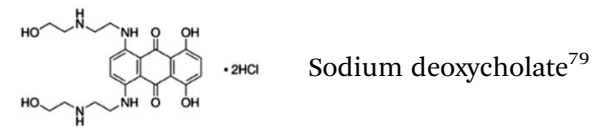

Mtb8.4

Protein, TB antigen, pI: $\quad$ Sodium docusate $\mathrm{e}^{174}$

6.3<smiles>C=CCN1CCc2ccc(O)c3c2C1(CO)C1CC(=O)C(C1)O3</smiles>

Sodium docusate $\mathrm{e}^{117}$

Naloxone

Naltrexone<smiles>O=C1CCCC23c4c(ccc(O)c4OC12)CCN3CC1CC1</smiles>

Sodium docusate ${ }^{117}$

Dextran sulfate ${ }^{92}$

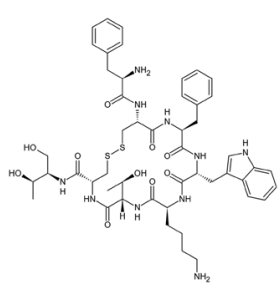

Ovalbumin (OVA) $43 \mathrm{kDa}$ protein, 385 residues, pI: 5.19

OZ439 mesylate (artefenomel)

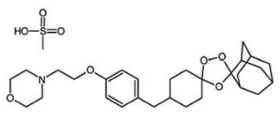

Papain

Pemetrexed

Polymyxin B

$$
23.4 \mathrm{kDa} \text { protein, } 212
$$$$
\text { residues, pI: 8.8-9.6 }
$$

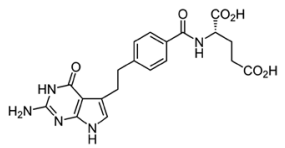

Cetrimonium bromide (CTAB) ${ }^{103}$

$N^{\alpha}$-Deoxycholyl-L-lysyl-methylester ${ }^{4}$

Oleic acid sodium salt ${ }^{78}$

Pamoic acid sodium salt ${ }^{78}$

Sodium dodecyl sulfate ${ }^{78}$

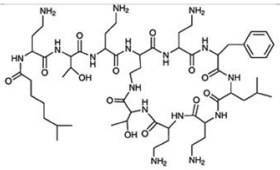

Sodium dodecyl benzenesulfonate ${ }^{78}$

Cetrimonium bromide (CTAB) ${ }^{39}$

Poly(inosinic acid)-poly (cytidylic acid) (poly(I : C))

Propranolol
Double-stranded RNA analog, TLR3 agonist<smiles>CC(C)NCC(O)COc1cccc2ccccc12</smiles>

Oleic acid $^{96}$

Sodium deoxycholate ${ }^{9,96}$

Sodium docusate ${ }^{9}$

Cetrimonium bromide (CTAB) ${ }^{39}$

Sodium oleate ${ }^{43,154}$

Sodium deoxycholate ${ }^{97}$
Sodium decanoate ${ }^{9,96}$

Sodium dodecyl sulfate ${ }^{92,96}$

Dextran sulfate ${ }^{61}$
Nanoprecipitation $^{79}$

PLG microspheres by emulsification ${ }^{174}$

PLA microparticles by precipitation with compressed antisolvent ${ }^{117}$

PLA microparticles by precipitation with compressed antisolvent ${ }^{117}$

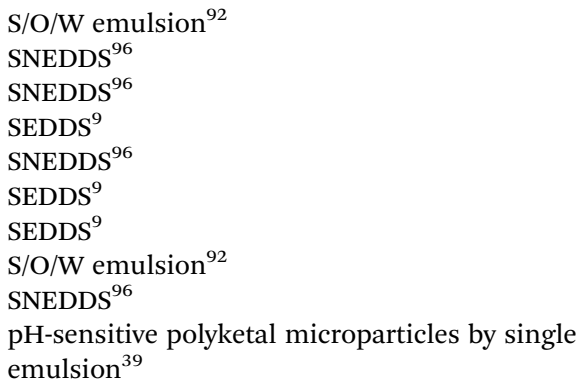

pH-sensitive polyketal microparticles by single emulsion $^{39}$

HPMCAS NPs by Flash NanoPrecipitation; in situ $\mathrm{HIP}^{43,154}$

SEDDS $^{97}$

Lyotropic liquid crystalline nanoparticles by homogenization (in situ $\mathrm{HIP})^{103}$

$\mathrm{W} / \mathrm{O} / \mathrm{W}$ emulsion ${ }^{4}$

PCL- $b$-PEG NPs by Flash NanoPrecipitation (FNP), in situ $\mathrm{HIP},{ }^{78}$ note: sodium decanoate, myristate, deoxycholate, 2-naphthalenesulfonate, 1heptanesulfonate, 1-octane-sulfonate, and 1decanesulfonate formed a precipitate when mixed with polymyxin B at $1: 1$ charge ratio but did not form NPs by FNP. Sodium hexanoate, benzenesulfonic acid, camphorsulfonic acid, and 1,2-ethanesulfonate did not form a precipitate when mixed with polymyxin $\mathrm{B}$ at $1: 1$ charge ratio. $\mathrm{pH}$-sensitive polyketal microparticles by single emulsion ${ }^{39}$

Alginic acid $^{61}$

Sodium acetate ${ }^{61}$

Sodium stearate ${ }^{61}$
Microemulsion by stearic acid coacervation ${ }^{61}$ 
Table 3 (Contd.)

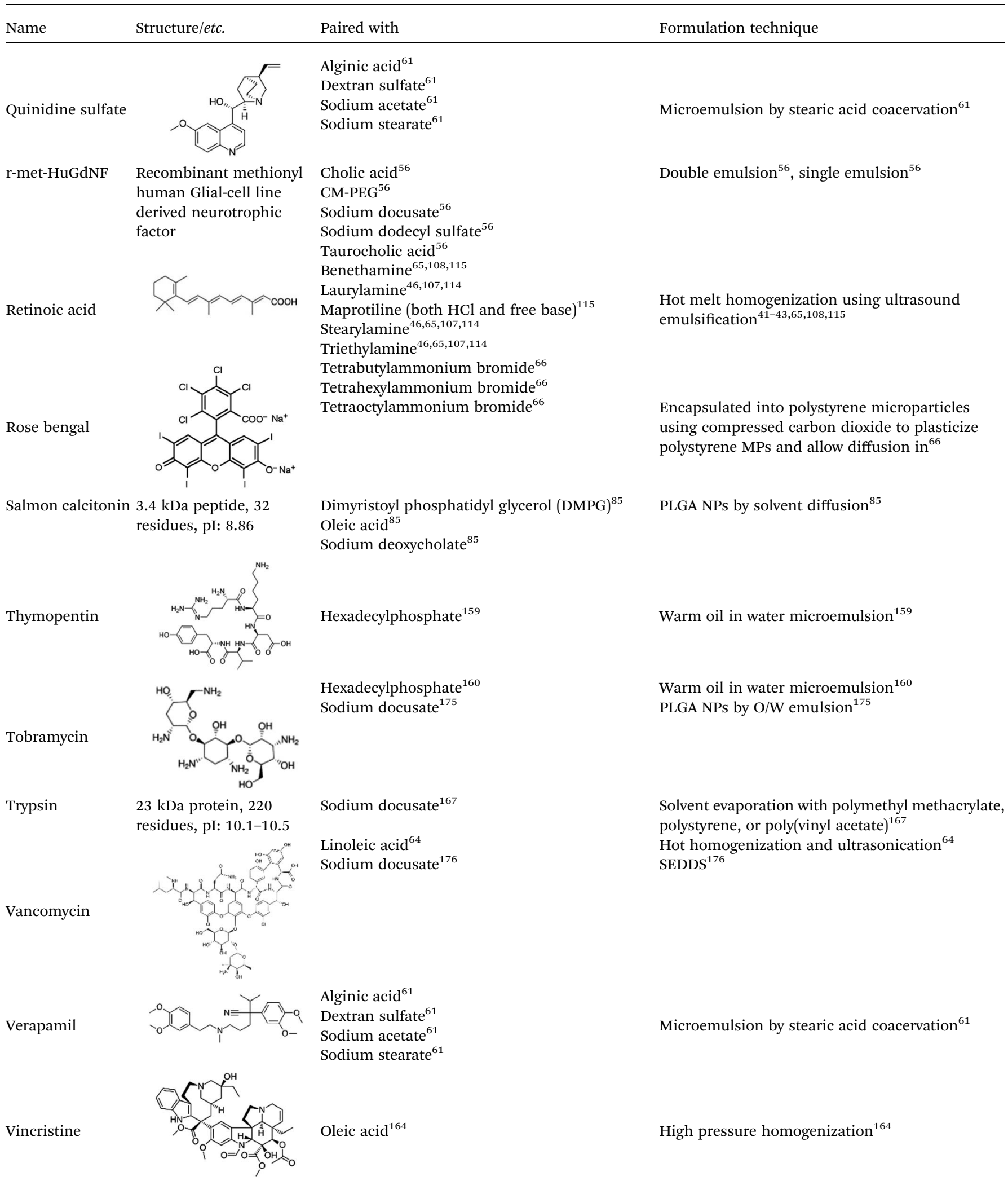

when one species is only partially ionized. Fig. 4 illustrates the $\mathrm{pH}$ window over which polymyxin $\mathrm{B}$ and oleic acid can be paired.
For peptide and protein drugs with many ionizable groups, the isoelectric point $\mathrm{pI}$ is a straightforward parameter to use, rather than trying to account for the $\mathrm{p} K_{\mathrm{a}}$ and ionizable state of each charged residue. As in charged polymers, the curve of 
Table 4 Examples of some polyvalent counterions used to encapsulate charged APIs. For a more complete survey of polyelectrolyte coacervation, see ref. 124-141

\begin{tabular}{llllll}
\hline Name & Structure & MW, Da & $\mathrm{p} K_{\mathrm{a}}$ & $\log P$ & Used to pair with: \\
\hline
\end{tabular}

Anions

Alginic acid (sodium alginate also used)
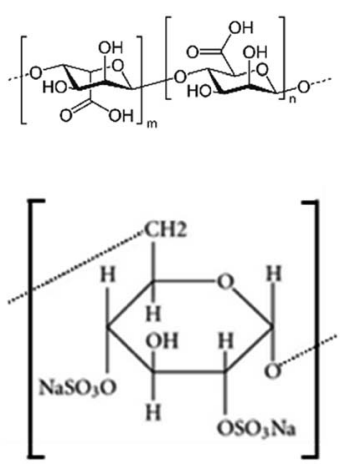

Dextran sulfate

Hyaluronic acid

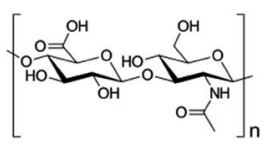

Cations

Chitosan

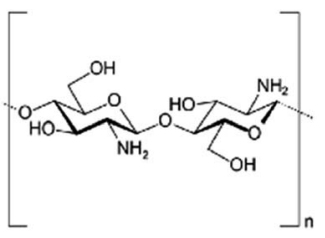

Doxorubicin ${ }^{61}$

Varies $\quad 1.5-3.5 \quad-1.5 \quad$ Propanolol $^{61}$

Quinidine sulfate $^{61}$

Verapamil $^{61}$

Bovine serum albumin ${ }^{91}$

Dalargin $^{104}$

Dexamethasone valine valine prodrug ${ }^{120}$

Doxorubicin $^{61,95}$

Idarubicin $^{95}$

IGG-Fab fragment ${ }^{90}$

Loperamide $^{104}$

Lysozyme ${ }^{59}$

Octreotide 92

Propanolol $^{61}$

Quinidine sulfate ${ }^{61}$

Verapamil $^{61}$

Varies $\quad 2.9 \quad-8.2 \quad$ Doxorubicin $^{153}$ charge versus $\mathrm{pH}$ for proteins is typically broader about the pI than an individual monomer would be. Curves denoting net charge versus $\mathrm{pH}$ are available for many proteins in the literature. ${ }^{67-69}$

When either the drug or counterion used has a carboxylic acid or non-quaternary amine head group, the resulting complex may demonstrate $\mathrm{pH}$-sensitive dissociation, which can be used to tune drug release. $\mathrm{pH}$-dependent release is useful in drug delivery, for example, for targeting to endosomes or tumors. Cationic peptides are popular in the HIP literature; these are positively charged at physiological and acidic pHs, so pH-dependent release could be accomplished by pairing them with fatty acids, rather than sulfates or phosphates. At a $\mathrm{pH}$ below the acid's $\mathrm{p} K_{\mathrm{a}}$, carboxylic acid become protonated, forming the uncharged free acid and decomplexing from their cationic counterparts. Hydrophobic and steric interactions from the former ion pair remain effective, but faster drug release can be expected. ${ }^{30,70-72}$ This will be discussed in more detail in the section on drug release.

Pinkerton et al. note that the $\mathrm{p} K_{\mathrm{a}}$ values of the two charged species should be different by at least two $\mathrm{pH}$ units for an ion pair to reliably form. Importantly, the authors pointed out that solvent quality affects $\mathrm{p} K_{\mathrm{a}}$ values. Therefore, when complexing in a mixed solvent of water and organics increasing the volume fraction of water may be useful to ensure complexation between

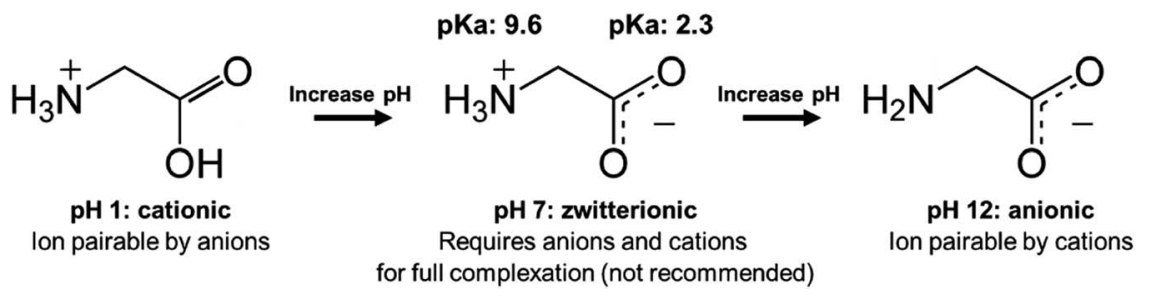

Fig. 3 Schematic illustrating the $\mathrm{pH}$-shifting strategy using glycine as a model API. At low $\mathrm{pH}$, carboxylic acid groups are protonated and uncharged. At high $\mathrm{pH}$, animes are deprotonated and uncharged. For some zwitterionic APIs, researchers have reported shifting $\mathrm{pH}$ to one extreme to turn off one type of charge prior to ion pairing. 


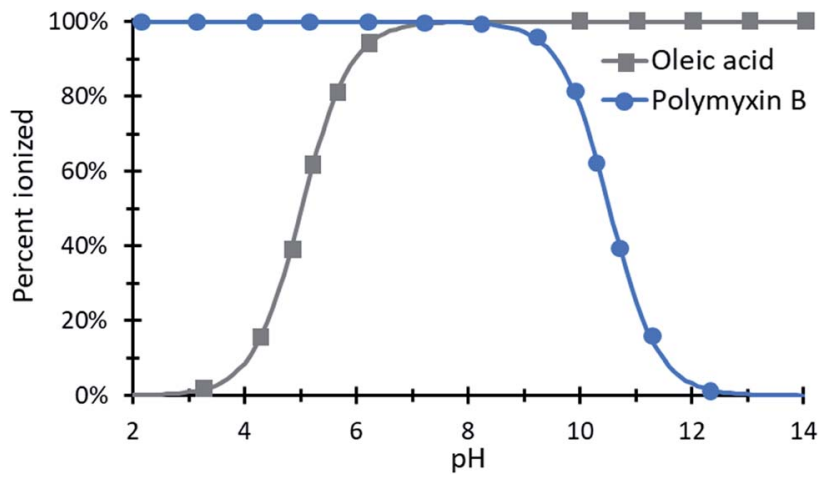

Fig. 4 An example species diagram showing the percent ionization of polymyxin $\mathrm{B}$ and oleic acid as a function of $\mathrm{pH}$. From approximately $\mathrm{pH}$ 6.5 to 9 , both species are nearly $100 \%$ ionized and could be paired with clear expectations about the resulting complex's charge ratio.

an anion and cation with $\mathrm{p} K_{\mathrm{a}}$ values close to neutral. ${ }^{47,73,74}$ Other researchers have noted that physical confinement, e.g. in a delivery vehicle, may affect $\mathrm{p} K_{\mathrm{a}}$ values as well; this phenomenon has the potential to affect HIP, and further study is needed ${ }^{75},{ }^{76}$.

An interesting study that to our knowledge has not been carried out in the literature would examine $\mathrm{pH}$-sensitive ion paired drug release behavior as a function of counterion head group. For example, Zupancic et al. paired cationic desmopressin with both sodium $n$-octadecyl sulfate and sodium stearate. ${ }^{77}$ The two have aliphatic tails of similar lengths, but the former has a sulfate head group and the latter has a carboxylic acid. If paired with a cationic API and encapsulated (ceteris paribus, and in a system with no other ionic or $\mathrm{pH}$-sensitive components), we would expect the $n$-octadecyl sulfate system's release profile not to vary between $\mathrm{pH}$ values of e.g. 6.5, 4.5, and 2.5. The system containing stearate should release differently at the three $\mathrm{pH}$ values, since stearate's $\mathrm{p} K_{\mathrm{a}}$ is 4.7 . Zupancic et al. found that sodium docusate and sodium oleate complexed with and precipitated desmopressin more effectively than either stearate or $n$-octadecyl sulfate, so the latter two counterions were not examined further. Both counterions must effectively complex with and precipitate the drug of interest. It is possible that desmopressin (1.1 kDa, 1 cationic charge) has too low of a charge density for the experiment proposed above.

We have discussed counterion $\mathrm{p} K_{\mathrm{a}}$ and $\log P$ values independently in the previous two sections. Researchers have noted that for a given counterion, it is prudent to also consider $\mathrm{p} K_{\mathrm{a}}$ and $\log P$ together. ${ }^{56,65,78}$ Carneiro et al. noted that triethylamine was a worse hydrophobic counterion for pairing with all-trans retinoic acid than both benethamine and stearylamine. Although triethylamine is a stronger base than the other two counterions, and should therefore be able to interact more easily with retinoic acid, it is so much less hydrophobic that the resulting complex does not have the desired lipophilicity. ${ }^{\mathbf{6 5}}$ Likewise, Lu et al. screened fifteen counterions as candidates to form hydrophobic complexes with the pentacationic peptide polymyxin $\mathrm{b} .{ }^{78}$ We found that at constant counterion $\mathrm{p} K_{\mathrm{a}}$, the threshold $\log P$ required to form an ion pair hydrophobic

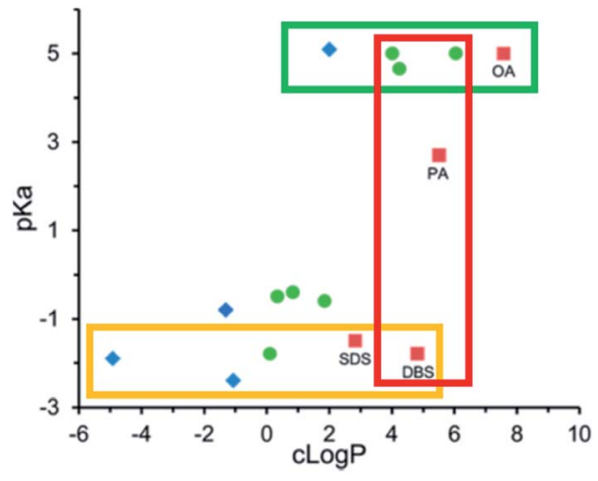

Fig. $5 \mathrm{p} K_{\mathrm{a}}$ and $\log P$ values for various anionic counterions. Complexes were pre-formed in $M Q$ water at a $1: 1$ charge ratio with polymyxin b. Blue diamonds indicate no precipitate was observed; green circles indicate a precipitate was observed, but was insufficiently hydrophobic for nanoprecipitation; and red boxes indicate a sufficiently hydrophobic precipitate was formed. Adapted with permission from H. Lu, P. Rummaneethorn, K. Ristroph, and R. K. Prud'homme, Hydrophobic Ion Pairing of Peptide Antibiotics for Processing into Controlled Release Nanocarrier Formulations, Mol. Pharmaceutics, 2018, 15(1), 216-225. Copyright (2017) American Chemical Society. ${ }^{78}$

enough for their encapsulation method (nanoprecipitation) varied. For aliphatic fatty acid sodium salts such as sodium hexanoate, sodium decanoate, and sodium oleate, precipitates formed at counterion $\log P$ values above 4 . Only sodium oleate, $\log P \sim 6.8$, formed complexes that precipitated as required for encapsulation (green box, Fig. 5). Sulfate surfactants formed sufficiently hydrophobic complexes at and above $\log P$ values of $\sim 2$ (yellow box, Fig. 5), suggesting that the sulfate surfactants interact more strongly with polymyxin b's cationic charges and form an ion pair more readily than the carboxylic acids. At a counterion $\log P$ of 5 , dodecylbenzene sulfate formed a sufficiently hydrophobic complex, but fatty acids decanoate and myristate did not (red box, Fig. 5).

We recommend that researchers complex their drug of interest using a suite of counterions at first, noting the $\mathrm{p} K_{\mathrm{a}}$ and $\log P$ values of the counterions used. The resulting complex's aqueous solubility and/or lipophilicity can be measured, and counterion chemistry or charge ratio can be varied to tune these values as desired.

Complexation: pre-formed vs. in situ. Ion paired complexes may be formed either prior to or during encapsulation; we call the former a 'pre-formed' complex and the latter an 'in situ' complex. In the literature, the vast majority of complexes are pre-formed in water or a water-organic mixture, then isolated by precipitation or filtration, washed, and dried. ${ }^{39,49}$ This approach allows researchers to measure the complex's $\log P$ empirically and fully characterize it using techniques such as differential scanning calorimetry (DSC), X-ray diffraction (XRD), NMR, FTIR, etc. ${ }^{79}$ Isolated complexes are often loaded into an oil phase or organic solvent (e.g. $\mathrm{DCM}^{\mathbf{8 0 , 8 1}}$ or acetone ${ }^{82}$ ) and treated as a lipophilic molecule. Since the oils and organics used are aprotic and often nonpolar, dissociation is unlikely.

Pre-formed complexes have a known stoichiometry and are already paired together, meaning electrostatic interactions between the drug and other delivery vehicle components are 
less likely to occur during encapsulation. This is particularly advantageous when encapsulation relies on charged species, such as lipid $\mathrm{pH}$ coacervation to encapsulate a complex. In lipid coacervation, ionized lipids are precipitated by dropping the solution $\mathrm{pH}$ below their $\mathrm{p} K_{\mathrm{a}}$ values. ${ }^{83,84}$ An undissolved hydrophobic pre-formed complex is less likely to ion pair with the lipids used than a dissolved, charged drug would be.

Many researchers have noted that when pre-forming at drug : surfactant charge ratios above $1: 1$, excess surfactants form micelles..$^{50,85-88} \mathrm{~A}$ solution that is cloudy and has visible precipitates at a $1: 1$ charge ratio may become clear when more surfactant is added, indicating the presence of micelles that solubilize the hydrophobic complex. When the $\log P$ of these micelle-loaded complexes is measured, it is unsurprisingly lower than the complex alone. ${ }^{50,61}$ For this reason, many studies using the pre-forming approach have stayed at or near a $1: 1$ drug : surfactant charge ratio to avoid micelles. Using higher charge ratios (e.g. $1: 2 \mathrm{drug}$ : surfactant, $1: 4$, etc.) should not be fully ruled out, though. Drying the pre-formed complex by lyophilization should disrupt micelles and yield a complex with a stoichiometry closer to the desired charge ratio, which may be required to tune release. This will be discussed further in the following section.

In situ ion pair formation is less common but avoids the micellization problem. Ashton et al. and Song et al. successfully paired AZD2811 with anionic surfactants during their nanoemulsion's formation, and Mussi et al. added docosahexaenoic acid to the oily phase of their SLN emulsion to pair with doxorubicin in situ. ${ }^{38,70,89}$ Pinkerton et al. and Lu et al. complexed small molecule and peptide APIs with counterions during rapid mixing in nanoprecipitation. ${ }^{43,47,78}$ A comparison between preformed and in situ ion pairs at a 1:1 charge ratio found no appreciable difference in the size of NPs formed by nanoprecipitation. ${ }^{78}$

Charge ratio. Screening multiple drug: counterion molar ratios is a straightforward series of experiments to perform. By doing so, researchers have measured how a number of important parameters vary with charge ratio: complexation efficiency, ${ }^{18,51,57,86-88,90-98}$ complex $\log P^{50,98-100}$ and zeta potential, ${ }^{93,101,102}$ drug encapsulation efficiency, ${ }^{30}$ and even droplet size in a SEDDS (self-emulsifying drug delivery system). ${ }^{94}$ Complexation efficiency in water is typically measured by centrifuging precipitated complexes and measuring the amount of free drug in the supernatant. When measured this way, efficiency is often reported as going through a maximum near a 1:1 drug : counterion charge ratio because of the solubilization of drug into micelles at higher ratios (more counterion) and insufficient complexation at lower ratios (less counterion). We note again that at the higher charge ratios, complex formation is not less efficient than at the $1: 1$, but that solubilization into micelles at equilibrium results in less complex settling during centrifugation. The final solution contains solubilized drug in thermodynamically stable micelles, and a second phase which is the drug : counterion complex with a different stoichiometry.

$\log P$ measurements as a function of charge ratio are sometimes reported to go through a maximum around $1: 1$ as well.
This is seen particularly if the experiment conducted involved forming an ion pair in water and then adding octanol. ${ }^{50}$ At charge ratios with higher counterion concentrations, micelles will have already formed in water by the time octanol is added and will be very unlikely to partition into the octanol phase. A better experimental design is to dissolve counterions first in octanol-saturated water and then add the drug of interest, ${ }^{99}$ or to dry the pre-formed complex before adding it into an octanolwater system. ${ }^{86,98}$ In this case, $\log P$ vs. charge ratio shows asymptotic behavior at higher counterion charge ratios.

Drug release as a function of drug : counterion charge ratio has also been reported..$^{71,78,103}$ As may be expected, at charge ratios with more equivalents of counterion, the release rate of drug from delivery vehicle slows. This is likely because the complex is more hydrophobic, or slower to dissociate, or both. We will discuss this phenomenon in more detail in the following section.

\section{Encapsulation techniques}

A number of common encapsulation techniques have been applied to molecules during or after hydrophobic ion pairing. This section discusses specific considerations that should be made when using these techniques to encapsulate an ion paired molecule. A general overview of some processing parameters and important outcomes such as encapsulation efficiency and drug loading is provided.

Encapsulation efficiency (EE): the encapsulation efficiency for a given drug formulation is calculated by measuring the amount of free drug (i.e. not encapsulated in or associated with delivery vehicles) after complexation and encapsulation. Separating free drug from delivery vehicles may be done via ultrafiltration or centrifugation, when the free drug and delivery vehicles have very different sizes, or by a technique such as size exclusion chromatography, for separating proteins from nanoparticles where filtration is ineffective. Once the amount of free API is measured, encapsulation efficiency is reported according to the following equation:

$$
\% \mathrm{EE}=100 \times\left(1-\frac{\text { mass of unencapsulated API }}{\text { total mass of API }}\right)
$$

For a formulation technique to be implemented at the industrial scale, high encapsulation efficiency - i.e. less material lost during processing - is desirable. High drug loading and few unit operations are also preferred.

Many papers report that HIP enabled researchers to encapsulate molecules that they previously could not, or that the technique improved their system's encapsulation efficiency, sometimes by more than $50 \%{ }^{18,49,84,87,88,90,101,104-110}$ Encapsulation efficiencies higher than $90 \%{ }^{49,60,84,102,103,105,106,111}$ and as high as $100 \%{ }^{57,78,82,109,112}$ have been reported.

Drug loading: the mass fraction of API in a nanodelivery vehicle is the drug loading. The rest of the vehicle mass consists of excipients such as lipids, stabilizing polymers, oils, etc. Using HIP, drug loadings ranging from $3-7 \%,{ }^{85,106,12} 10-20 \%,{ }^{57,81,101}$ and up to $30 \%$ (ref. 47,78 and 113) have been reported. Some formulation 
strategies have inherent limits on drug loading; for example, $\mathrm{S} / \mathrm{O} /$ $\mathrm{W}$ emulsions form percolation networks when the oil phase containing a hydrophobic drug reaches too high a volume fraction within a single droplet. ${ }^{12}$ Therefore, in these systems there is an inverse relationship between drug loading and vehicle stability, which is undesirable at scale and for clinical application.

\subsection{Emulsions}

Single (e.g. oil-in-water, $\mathrm{O} / \mathrm{W})^{38,39,56}$ and double (e.g. solid-in-oilin-water or water-in-oil-in-water, $\mathrm{S} / \mathrm{O} / \mathrm{W}$ or $\mathrm{W} / \mathrm{O} / \mathrm{W}),{ }^{18,90,91}$ etc. see Table 3 emulsions have been used to encapsulate ion paired complexes into droplets that may then be dried or otherwise further processed. Both require surfactants to stabilize, and typically a non-ionic species such as PVA is used. Using an ionic surfactant as an emulsion stabilizer may interfere with the hydrophobic complex's formation or stability.

Researchers commonly pre-form hydrophobic complexes prior to introducing them into an emulsified system. This has an advantage over in situ formation in that the complex may be added to the oil phase before emulsification, which promotes better encapsulation. If the hydrophilic component were introduced in the aqueous phase and the hydrophobic counterion were introduced via the oil phase, pairing would likely occur at oil-water interfaces if at all (and the degree of counterion ionization in the oil phase would be difficult to determine and control). If both components were added unpaired in a mixed oil phase, pairing would again be limited by ionization. Finally, if API and counterion were introduced in water and allowed to pair, the resulting hydrophobic complex would need to partition into the oil droplets, which would take longer and be less efficient than loading the pre-formed complex into oil, where it will prefer to remain.

A HIP complex's final geometry and possible amphiphilicity are another parameter that should be considered. A hydrophilic API's water-soluble charged group may be complexed by HIP, but other polar regions on the molecule may result in an amphiphilic complex that may tend to accumulate on the emulsion droplets' oil-water interface. ${ }^{97}$ Using excess counterion, or a counterion with larger hydrophobic regions, may partially mitigate this effect. Proteins may be stabilized from denaturation at oil-water interfaces may be stabilized via ion pairing if they are complexed in such a way that their tertiary structure is largely preserved and their hydrophilic regions, which would lead to interfacial aggregation if exposed, are hidden..$^{12,100}$

\subsection{Lipid nanoparticles}

Hydrophobic complexes have been incorporated into solid lipid nanoparticles either by emulsification from a hot

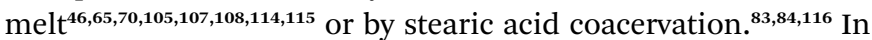
the former, non-ionic lipids and surfactants such as glyceryl behenate were heated and added to an oil phase along with a pre-formed complex. The hot oily phase was added to water and sonicated. With no other charged species present, it is unlikely that the ion pair was disrupted prior to encapsulation. Carneiro et al. note that without complexation, the API of interest, all-trans retinoic acid, resides primarily at the lipid- water interface, and that hydrophobic complexation helps incorporate it more fully in the lipid matrix. ${ }^{65}$

Lipid nanoparticle formation by stearic acid coacervation involves lowering the $\mathrm{pH}$ of a solution of water and ethanol containing stearic acid to protonate and precipitate it as a free acid..$^{\mathbf{8 3 8 4 1 1 6}}$ In these systems, a pre-formed hydrophobic complex was added along with ethanol into the hot aqueous solution of stearic acid. Since ion pair formation and stability vary with ionic strength and $\mathrm{pH}$, it remains unknown if the ion pair remained together during this formulation strategy, or if dissociation (and possibly re-pairing between the drug and stearic acid, before the $\mathrm{pH}$ dropped too low) occurred. Therefore the final stoichiometry and identity of the ion pair are difficult to know, even using a pre-formed system in the presence of additional potential ion pairing partners.

\subsection{Precipitation}

Controlled nanoprecipitation techniques such as Flash NanoPrecipitation take advantage of diffusion-limited aggregation between precipitating molecules in an aqueous or mixed solvent system. Hydrophobic complexes are well-suited for this approach, since their water solubility is very poor and they precipitate quickly. Rapid precipitation followed by stabilization, e.g. surface deposition of the hydrophobic block of a block copolymer, yields kinetically trapped core-shell nanoparticles. ${ }^{47}$ Rapid, good mixing will result in homogeneous nucleation and growth, which is desirable in nanoprecipitation. Heterogeneous nucleation or poor mixing may allow sufficient time for the formation of a thermodynamically favoured micelle phase from excess hydrophobic counterion. This is undesirable because the hydrophobic polymers or polymer blocks used in nanoprecipitation may not deposit onto a micelle's charged surface as they would onto a hydrophobic surface, and the same kinetically-trapped particle may not be formed.

Researchers have demonstrated both in situ and pre-formed ion pairing approaches with nanoprecipitation. Water is typically used as an antisolvent to induce precipitation, so using salt forms of the API is a straightforward method of ensuring an initially ionized state of the API. This means that in situ complex formation, followed immediately by precipitation, is easy to accomplish. Unlike the water-oil systems such as those used for emulsions, nanoprecipitation systems use water-miscible organic solvents, meaning interfacial partitioning is not a factor. The main limit to complexation is therefore diffusion, falling in line with nanoprecipitation's typical diffusion-limited aggregation kinetics. Lu et al. reported that even in a Flash NanoPrecipitation system, where rapid mixing on the order of $2 \mathrm{~ms}$ is followed by nucleation, growth, and stabilization by block copolymer adsorption all within about $20 \mathrm{~ms}$, complexes formed in situ were efficiently encapsulated..$^{\mathbf{4 3} 78}$ This suggests the time scale of complexation and precipitation is less than $20 \mathrm{~ms}$. This is comparable to the precipitation time of a strongly hydrophobic $(\log P>5)$ molecule or pre-formed hydrophobic complex - in the same system.

Precipitation with a compressed antisolvent (PCA) has also been used to encapsulate HIP complexes into nanoparticles or microparticles. ${ }^{\mathbf{8 2 1 1 7 - 1 1 9}}$ Because the mixing in PCA is between 
a solvent containing the drug of interest and a chamber of pressurized gas, pre-formation of the hydrophobic complex is required.

\subsection{Others}

Other formulation strategies can be viewed through a similar lens to the one we have used above. Techniques that treat a preformed complex such as a typical hydrophobic molecule are valid provided they have not neglected the complex's sensitivity to salts and $\mathrm{pH}$. For example, self (micro/nano)-emulsifying drug delivery systems (S[M/N]EDDS) may use either ionic or non-ionic surfactants. We described when discussing lipid nanoparticles that ionic surfactants could disrupt ion pairing or exchange with a complex's counterions - these considerations are important to keep in mind when modifying a system usually used to encapsulate a non-ionic hydrophobic molecule to one capable of encapsulating a HIP complex.

Systems that require $\mathrm{pH}$ modulation are not wholly ineligible for use with HIP, but care should be taken to ensure that the complex is not disrupted if possible. Consider Iqbal et al., who used a unique method of interpolymer complexation between polyethylene glycol and poly(acrylic acid) to form nanoparticles. ${ }^{11}$ This required adjusting the solution $\mathrm{pH}$ to 3 to protonate poly(acrylic acid); the cationic drug of interest was pre-formed with docusate and introduced along with Pluronic F68 in ethanol into an acidic aqueous PAA solution. Docusate has a sulfonate head group that should remain charged at $\mathrm{pH} 3$, and PAA was already protonated and uncharged before the complex was added. Taken together, these suggest the pre-formed leuprolide:docusate complex was likely to survive intact in this formulation technique than in (1) one where it encountered another ionized species (as in the case of stearic acid coacervation discussed above) or (2) a system using a hydrophobic counterion (e.g. a fatty acid) that would be deprotonated at the final $\mathrm{pH}$.

\section{Ion paired drug release from a delivery vehicle}

Drug release from a delivery vehicle containing a hydrophobic complex varies with the type of vehicle (core-shell nanoparticle, SLN, double emulsion, SEDDS, etc.), but useful similarities exist. The ion paired drug will behave like a hydrophobic molecule as long as it remains complexed. Once complexation is reversed, the original hydrophilic molecule and hydrophobic counterion are regenerated and will usually partition out of the delivery vehicle. De-complexation is driven by one of two main mechanisms: counterion competition by salts or $\mathrm{pH}$-driven charge negation. The former occurs when salts in the surrounding medium are able to access the complex and outcompete the hydrophobic ion pair, leading to dissociation. The high ionic strength in the surrounding medium screens the charges between the two regenerated species, so recomplexation is unlikely. The former follows a similar mechanism, protonating or deprotonating one of the charged species and leading to de-complexation.

Both mechanisms depend on water accessing the hydrophobic complex. For this reason, the vehicle's type and geometry are both important. Core-shell nanoparticles have a less water-accessible core than PLGA-stabilized double emulsions, for example, and it may be expected that they release drugs more slowly in similar salt/pH conditions. Because water can access ion pairs at the water-vehicle interface much more readily than complexes deep in the vehicle core, release is expected to occur from the outside in. After ion pairs at the surface have been de-complexed and partition into the bulk phase, water will be able to access the complexes deeper in the vehicle. During this 'erosion,' the vehicle itself may lose structural integrity or collapse. ${ }^{61}$ For vehicles with low drug loading, the hydrophobic complex's location in the vehicle is another factor to consider. A complex with amphiphilic character that resides primarily on the vehicle surface is easily water-accessible, and rapid burst release may be observed. ${ }^{65,97,111}$

The fact that de-complexation is a precursor to this type of release explains why slower drug release is seen at higher charge ratios. ${ }^{71,78,103}$ A monovalent drug complexed with a single monovalent counterion should fully dissociate much more quickly than a drug complexed with four, and will not release from the vehicle until it is fully dissociated. In the $1: 4$ charge ratio case, only one of the counterions can truly form an ion pair with the drug (see the preceding section on drug : counterion charge ratio). The remaining three counterions can remain associated with the complex, though, adsorbing onto the first counterion via tail-tail hydrophobic interactions. The resulting large hydrophobic surface area serves as a mass transfer barrier that slows water diffusion to the site of ion pairing. For this reason, it is more difficult for water to access and dissociate the $1: 4$ complex than the $1: 1$ complex, so drug release is slower. The probability of a drug re-complexing with a hydrophobic counterion after salt-driven decomplexation also increases with the number of hydrophobic counterions near a drug molecule.

After dissociation, the therapeutic and counterion are regenerated. Without its hydrophobic counterion, the therapeutic is likely too hydrophilic to remain associated with the vehicle (even if the solution $\mathrm{pH}$ has turned off the drug's charge) and will partition into the bulk. Depending on its chemistry and the bulk $\mathrm{pH}$, the counterion may either diffuse into the bulk or remain with the vehicle. For example, we found that at a $1: 4$ polymyxin B:oleate charge ratio, drug release plateaued around $35 \%$. This suggests that after soluble polymyxin b was released, the poorly-water-soluble oleate fatty acids remain with the nanoparticle and may form an oleate/oleic acid liquid crystal phase in or around the NP core. This type of plateauing release profile was not observed for polymyxin paired at a $1: 4$ polymyxin:SDS charge ratio, because SDS is more watersoluble and prefers to partition into the bulk. ${ }^{78}$

Complexation formation, dissociation, and release have all been found to be a function of bulk ionic strength. ${ }^{18,38,44,45,72,120}$ As expected, at higher ionic strength, it is more difficult to form complexes due to charge screening, and, if formed, complexes dissociate and drugs are released faster at higher salt concentrations. PBS, sodium chloride, and serum are common release media. Researchers have found both complexation ${ }^{18,57,120,121}$ and release $\mathrm{e}^{30,38,48,70-72,105,112,121}$ to be $\mathrm{pH}$-dependent. These assays are 
usually run at $\mathrm{pH}$ values at and below 7.3. In most cases, faster release at lower $\mathrm{pH}$ has been observed as expected.

This understanding of the mechanism behind salt- and $\mathrm{pH}-$ dependent release is useful. Many nano-scale delivery vehicles are stable in water and can be stored in deionized water without beginning to release their payload. For parenteral or oral formulations, the body's natural ionic strength will trigger release. $\mathrm{pH}$-dependent release could be useful for targeting to endosomes, tumors, or different regions in the intestinal tract. On the other hand, $\mathrm{pH}$ sensitivity may rule out some long-term depot delivery strategies; for example, it has been welldocumented that PLGA microparticles may acidify as the polymer is degraded over time. ${ }^{\mathbf{1 2 , 1 2 2}}$ An acid microenvironment could trigger ion pair dissociation and lead to faster release.

The previous several paragraphs have discussed drug release following ion pair dissociation. In general, unless a hydrophobic complex is exposed to salts or $\mathrm{pH}$ changes, it should behave like a hydrophobic molecule - depending on the vehicle and the chemistry of the species that make up the pair, the complex could still be released intact from the vehicle. Hydrophobic molecules in nano-scale delivery vehicles can diffuse and, depending on their solubility in the release medium, may still partition into the bulk. The complex's size and hydrophobic interactions with the delivery vehicle are barriers to diffusion. ${ }^{65,66,116}$ When buffers containing only salts are used as simple release media, this type of release is unlikely. In more complex, more realistic release media - i.e. those containing some kind of hydrophobic sink such as albumins or bile salt micelles - this type of direct release, as well as salt- or pH-driven release, may occur simultaneously. The driving forces for

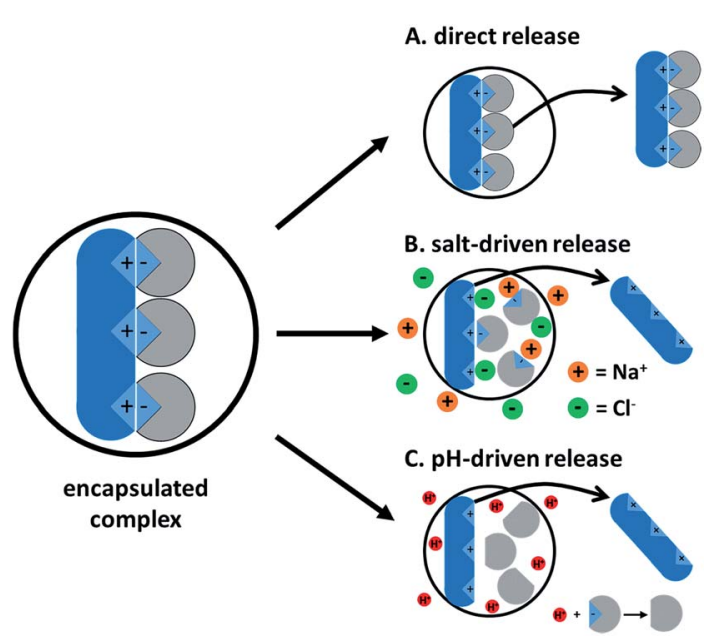

Fig. 6 A summary of how complexed drugs may be released from a delivery vehicle. (A) The intact complex may release directly from the particle into the bulk. This type of release is a function of the complex's solubility in the bulk, so hydrophobic sinks such as bile salt micelles or albumins in the bulk phase will provide more of a driving force than simply a buffer. (B) Salts lead to counterion competition and decomplexation, which is followed by release as the water-soluble drug is released into the bulk. (C) When counterions such as fatty acids are used, lowering the $\mathrm{pH}$ below their $\mathrm{p} K_{\mathrm{a}}$ will lead to protonation. The protonated counterions will no longer complex the drug, leading to release as in (B). diffusion of all species, including paired and unpaired drugs and paired, unpaired, and uncharged counterions, should be considered.

It is straightforward to see that both major exit routes from a particle - either following dissociation or as an intact complex - depend on the complex itself and the counterion used. Alkyl tail length or hydrophobic group size, for example, affect both. In the case of post-dissociation release, larger hydrophobic surface areas decrease water permeability and slow decomplexation, as discussed in the paragraph about release as a function of charge ratio. And in the case of release as an intact complex, longer alkyl tails both decrease the complex's solubility in the bulk and increase steric and hydrophobic interactions that tend to keep the complex in its vehicle. As counterion hydrophobicity increases, therefore, release tends to decrease (Fig. 6). ${ }^{29,38,65,78,88}$

\section{Bridging polyelectrolyte coacervation, polymer-surfactant complexation, and hydrophobic ion pairing}

The HIP literature has generally not overlapped with the literature from the fields of polyelectrolyte-polyelectrolyte coacervation or polyelectrolyte-surfactant complexation. The fields share a number of similarities that we will highlight here. Polyelectrolyte complex coacervation used here refers to the phase separation induced when oppositely-charged polyelectrolytes or ionomers ion pair with one another, and is not to be confused with the acid-induced lipid precipitation technique mentioned earlier (which is also called 'coacervation'). Polyelectrolyte-surfactant complexation has been studied extensively and is useful in a number of industrial applications, including personal care products and detergents. ${ }^{\mathbf{1 2 3}}$

\subsection{Polyelectrolyte coacervation}

Ion pairing between polyelectrolyte species results in the formation of a highly electrostatically crosslinked complex that phase separates from its surrounding media; the new phase may be either a solid ('precipitate') or liquid ('coacervate'), but the term 'complex coacervation' is applied in both cases. ${ }^{\mathbf{1 2 4 - 1 2 6}}$ Stoichiometric ratios (i.e. $1: 1$ cation : anion) are common in this literature, since these systems tend to form more distinct coacervate phases from charge neutral conditions - at uneven ratios, electrostatically-stabilized colloidal particles may form. ${ }^{125}$ Like hydrophobic ion pairs, coacervate phases are sensitive to salt and may be dissociated at sufficient ionic strength or as a function of $\mathrm{pH}$, depending on the chemistry.

The entropic and enthalpic effects of coacervate formation and phase separation have been studied and reported elsewhere. ${ }^{125,127-132}$ Interestingly, polymers in a complex coacervate may remain mobile, and rearrangement is possible. Many studies focus on the coacervate's phase behaviour ${ }^{\mathbf{1 3 3}}$ or rheological ${ }^{\mathbf{1 3 4}}$ or thermal ${ }^{\mathbf{1 3 5}}$ properties. The final phase's $\log P$ (and hydrophobicity in general) is not a major concern in complex 
coacervation, since the mechanism of phase separation is different and the ultimate goal of coacervation is not necessarily to increase hydrophobicity or modify solubility as in HIP.

Because complex coacervates form distinct phases, the technique has been studied as a possible method of encapsulating polyvalent drugs such as peptides and proteins. ${ }^{136-139}$ For insufficiently charged proteins, 'supercharging' - adding more charged amino acids - increases charge density and can lead to more reliable coacervation. ${ }^{140}$ Some similarities and differences between encapsulation using coacervation and HIP may be seen. Encapsulation via coacervation is typically carried out near or at a stoichiometric charge ratio. ${ }^{136,138}$ This differs from the HIP literature, where a screen of different drug : counterion charge ratios is commonly performed and the resulting complex's $\log P$ is characterized. Using a complex coacervate as a delivery vehicle introduces problems similar to $\mathrm{W} / \mathrm{O} / \mathrm{W}$ double emulsions; namely, a tradeoff between drug loading and encapsulation efficiency. ${ }^{141}$ Another notable difference between HIP and PE coacervation other than the molecular weights of the species used is the lack of ionic crosslinking in much of the HIP literature. Most HIP studies use monovalent small molecule counterions (exceptions are discussed in the following paragraph), but PE coacervation depends on polyvalency to form its characteristic crosslinked polymer networks.

Some papers using small-molecule surfactants for HIP have also used polyvalent counterions to complex drugs, and most unfortunately fail to make the distinction between the two techniques in their descriptions of what was done. The most common polyvalent counterion in these cases is dextran sulfate, a polyanionic sugar that does not contain distinct hydrophobic domains..$^{59,91,92,104,120}$ When it is used to complex multivalent APIs such as proteins, dextran sulfate forms a complex coacervate rather than a true hydrophobic ion pair. The coacervate may still be encapsulated using methods that also encapsulate hydrophobic ion pairs, but drug release from ion pairs compared to coacervates may differ noticeably. Consider Song et al., who found that drug release of an ion paired multivalent small molecule AZD2811 varied significantly whether using pamoic acid (divalent) or xinafoic acid (monovalent, effectively half of pamoic acid) at the same charge ratio. ${ }^{38}$ The difference in release profile observed was most likely a function of crosslinking between divalent pamoic acid and the multivalent small molecule, which resulted in slower release. It is easy to see that a system containing dextran sulfate, which has much greater protein crosslinking potential than divalent pamoic acid, could be expected to have very different release kinetics from a system using a small molecule surfactant counterion. Understanding and carefully distinguishing between the two approaches will be beneficial for future studies aiming to ionically complex and encapsulate charged therapeutics.

\subsection{Polymer-surfactant complexation}

Polymer-surfactant complexation and phase behavior have been studied for decades. ${ }^{142,143}$ The field has carefully examined precipitate formation as a function of many parameters familiar to the hydrophobic ion pairing literature, including charge density, ${ }^{144}$ stoichiometry, and formation and dissociation in the presence of salts. ${ }^{145}$ Studies of self-assembly have developed binding isotherms and a thermodynamic understanding of surfactant monolayer and bilayer formation. ${ }^{123,146,147}$ Important questions in this field include: what are the phase behavior and rheological properties (surface tension, viscosity, etc.) of polymer-surfactant complexes? ${ }^{144,148,149}$ Do they form one-phase or two-phase systems, and does this change upon dilution - i.e., what are the critical aggregation concentrations? What is the effect of surfactant alkyl tail length on this behavior? ${ }^{146,150}$ Are the polymers binding to surfactant monomers, or micelles? ${ }^{145,151}$

The polymers used in these studies often have higher molecular weight and more regular charge spacing than the peptides and proteins complexed with surfactants in the HIP literature. As in the coacervation literature, imparting additional hydrophobicity to a polymer is not necessarily the ultimate goal of a study, and drug encapsulation is rarely discussed. These studies often examine wetting or solubilization behavior, and are useful in the development of new detergents and shampoos.

A familiarity with the field of polyelectrolyte-surfactant complexation will help hydrophobic ion pairing researchers appreciate similar sensitivities - e.g. to salts, temperature, or $\mathrm{pH}$ - that their own systems might experience. It will also help them develop an appreciation for surfactant phase behavior, which has not been well-characterized in most studies that use HIP to encapsulate a drug molecule.

\section{Perspective}

Encapsulating hydrophilic therapeutics via hydrophobic ion pairing is a useful technique and offers a number of attractive possibilities. In this section, we highlight four major ones: (1) co-encapsulating hydrophilic and hydrophobic drugs, (2) forming a HIP complex from two therapeutic species, (3) decreasing API crystallinity, and (4) tuning drug release rates by simply altering the counterion used in HIP.

Many encapsulation techniques that have been optimized for hydrophobic therapeutics can easily be adapted to work on ion paired hydrophobic complexes. This introduces a unique and powerful possibility: straightforward co-encapsulation of hydrophilic and hydrophobic therapeutics into a single delivery vehicle at high loadings. This is highly desirable, and is not possible using encapsulation techniques developed specifically for water-soluble drugs, e.g. W/O/W emulsions. Using two different encapsulation techniques to prepare two populations of particles encapsulating two therapeutics can be difficult (and expensive), especially if the particle chemistries, sizes, and fates are intended to match. There can be no guarantee of simultaneous delivery or even co-delivery in the body using a mixed population of particles, particularly at the single-cell level where local concentrations of both species may be important for therapeutic synergy.

Using HIP, researchers have co-encapsulated hydrophilic and hydrophobic therapeutics. ${ }^{30,71,103}$ Tuning release rates of co- 
encapsulated drugs from these two classes is an exciting prospect for future study. For example, Zhou et al. found their hydrophobic therapeutic released at a $\mathrm{pH}$-independent rate, while the ion paired complex's release varied with $\mathrm{pH} .{ }^{30}$ Future formulations may take advantage of this difference in behaviour while still benefiting from co-localization. Or, if the mismatch is undesirable, researchers could tune parameters discussed above such as charge ratio, counterion chemistry, etc. to make hydrophilic and hydrophobic release rates match.

Another exciting opportunity of hydrophobic ion pairing is the co-delivery of two water-soluble charged APIs by ion pairing the two of them together. Denadai et al. formed a hydrophobic complex from two therapeutic small molecules, cationic chlorhexidine and anionic losartan, but did not encapsulate the result in a separate delivery vehicle. ${ }^{152}$ Other papers report choosing their counterion based on therapeutic synergy. Kalhapure et al. chose from among several similarly-lipophilic counterions - palmitoleic, oleic, linoleic, linolenic, and arachidonic acid - by checking the ability of each to inhibit $S$. aureus growth. Linoleic acid's MIC was superior to the others, so it was used to pair with vancomycin. ${ }^{64}$ Similarly Oliveira et al. ion paired doxorubicin with $\alpha$-tocopherol succinate to take advantage of the anticancer properties of each, ${ }^{\mathbf{1 0 5}}$ and Li et al. paired doxorubicin with hyaluronic acid since the latter is possibly useful for tumor targeting. ${ }^{153}$

Several papers have reported that ionic complexation with hydrophobic counterions formed an ion pair with lower crystallinity than its individual components. ${ }^{28-30,43,46,47,154}$ Improved amorphous character may be useful for quickly releasing drugs, particularly as intact complexes, if desired. It may also be helpful for formulating hydrophobic ionic drugs into stable NPs with faster dissolution kinetics. ${ }^{43}$ In this case, controls with a non-ionic analogue of the counterion (e.g. methyl esters instead of carboxylic acids; see Oliviera and Mussi for examples of this important control experiment) should be used to determine if reduced crystallinity is simply a co-core confinement effect or truly the result of HIP. ${ }^{70,105}$

For formulations containing a HIP complex, tuning drug release rates by changing the hydrophobic counterion or API : counterion charge ratio used is a straightforward and powerful tool for formulation scientists and drug delivery researchers. ${ }^{71,78,103}$ Changing the amount or type of counterion used during a formulation technique while holding all other excipients and processing steps constant is relatively easy and should allow for several formulations with different release rates - and therefore possibly different PK profiles - to be developed and tested in rapid succession.

Hydrophobic ion pairing remains an active area of research, and several fundamental aspects of the process remain unclear: the precise dynamics of API-counterion assembly, aggregation/ precipitation, and dissociation/release as a function of drug and surfactant chemistry (e.g. head group $/ \mathrm{p} K_{\mathrm{a}}$, charge density, charge ratio, $\mathrm{pH}$, ionic strength, hydrophobic moieties, etc.) are active areas of research. The phase behavior of surfactants used as hydrophobic counterions once paired is similarly unknown; this may prove to be an important parameter in modulating release. ${ }^{78}$ Molecular dynamics simulations may be the best way to examine the details of these phenomena, as they have been used extensively to study PE coacervation and PE-surfactant complexation. ${ }^{\mathbf{1 3 0 , 1 3 1}}$

We have reviewed how and why drug release from an ion paired system varies with counterion. It remains unknown if a mixture of counterions in a single formulation could lead to two different release profiles simultaneously; e.g. preparing two different pre-formed complexes of a cationic peptide with two different anionic surfactants, then incorporating both into a single formulation to achieve an initial burst release followed by slower, steady release over time.

This review has summarized the many proof-of-concept studies that demonstrate hydrophobic ion pairing as a useful tool for complexing and encapsulating hydrophilic therapeutics across several classes - small molecules, peptides, protein fragments, and full proteins such as antibodies and enzymes into nano-scale delivery vehicles. The technique is straightforward, uses accessible and inexpensive surfactants, and yields hydrophobic complexes that may be precipitated, emulsified, or otherwise packaged using existing technology. Fundamental questions remain about counterion phase behavior, the details of hydrophobic and ionic interactions during complexation, and the exact mechanism of drug release across several chemistries and external parameters. Moving forward, the field offers exciting possibilities such as co-encapsulation of hydrophobic and hydrophilic therapeutics, collaborations with the related fields of polyelectrolyte coacervation and polyelectrolytesurfactant complexation, or the co-encapsulation and codelivery of two ionic APIs by forming a HIP complex from the two of them.

\section{Abbreviations}

$\begin{array}{ll}\begin{array}{l}\text { a priori } \\ \text { API }\end{array} & \begin{array}{l}\text { Beforehand } \\ \text { ceteris } \\ \text { paribus }\end{array} \\ \text { CTAB } & \text { With all else being equal } \\ \text { DCM } & \text { Cetrimonium bromide } \\ \text { DSC } & \text { Dichloromethane } \\ \text { et al. } & \text { Differential scanning calorimetry } \\ \text { FDA } & \text { And the others } \\ \text { FTIR } & \text { United States Food and Drug Administration } \\ \text { HIP } & \text { Fourier-transform infrared spectroscopy } \\ \text { in situ } & \text { Hydrophobic ion pair/pairing } \\ \text { IP } & \text { on site/in place } \\ \text { NMR } & \text { Nuclear magnetic resonance } \\ \text { N/P ratio } & \text { Molar ratio of cationic nitrogen in lipids to anionic } \\ & \text { phosphorous in nucleic acids, used in the lipoplex } \\ & \text { literature } \\ \text { NCs } & \text { Nanocarriers } \\ \text { NPs } & \text { Nanoparticles } \\ \text { PBS } & \text { Phosphate-buffered saline } \\ \text { PLGA } & \text { Poly(lactic-co-glycolic acid) } \\ \text { SDS } & \text { Sodium dodecyl sulfate } \\ \text { SLN } & \text { Solid lipid nanoparticle } \\ & \end{array}$


S(M/N) Self-(micro/nano)emulsifying drug delivery system EDDS

XRD

X-ray diffraction

\section{Funding}

We acknowledge financial support from the Bill and Melinda Gates Foundation (BMGF OPP1150755), The Princeton SEAS Helen Shipley Hunt Fund, and the National Science Foundation for the research fellowship for K. D. R. (DGE-1656466) and (CBET 1605816).

\section{Conflicts of interest}

There are no conflicts to declare.

\section{Acknowledgements}

The authors thank Dr Nathalie Pinkerton, Dr Robert Pagels, Mr Chester Markwalter, and Mr Douglas Scott for intellectual discussion and for their help reviewing the document.

\section{References}

1 J. D. Meyer and M. C. Manning, Hydrophobic Ion Pairing: Altering the Solubility Properties of Biomolecules, Pharm. Res., 1998, 15(2), 188-193.

2 T. Wang and X. Wang, Solubilization of an indicator dye for optical fiber chemical sensors in supercritical $\mathrm{CO}_{2}$ by ion pairing, Sens. Actuators, B, 2003, 89(1), 144-149.

$3 \mathrm{~S}$. Wu, A. Buthe and P. Wang, Organic-soluble enzyme nanocomplexes formed by ion-pairing with surfactants, Methods Mol. Biol., 2011, 743, 51-63.

4 R. Pangeni, J. U. Choi, V. K. Panthi, Y. Byun and J. W. Park, Enhanced oral absorption of pemetrexed by ion-pairing complex formation with deoxycholic acid derivative and multiple nanoemulsion formulations: preparation, characterization, and in vivo oral bioavailability and anticancer effect, Int. J. Nanomed., 2018, 13, 3329-3351.

5 S. K. You, H. H. Kwon, J. M. Lee, S. C. Shin and C. W. Cho, Studies on the formation of hydrophobic ion-pairing complex of alendronate, Arch. Pharmacal Res., 2009, 32(7), 1055-1060.

6 T. N. Q. Phan, I. Shahzadi and A. Bernkop-Schnürch, Hydrophobic ion-pairs and lipid-based nanocarrier systems: the perfect match for delivery of BCS class 3 drugs, J. Controlled Release, 2019.

7 S. A. Megwa, S. E. Cross, M. W. Whitehouse, H. A. Benson and M. S. Roberts, Effect of ion pairing with alkylamines on the in-vitro dermal penetration and local tissue disposition of salicylates, J. Pharm. Pharmacol., 2000, 52(8), 929-940.

8 A. S. Torky, M. S. Freag, M. M. A. Nasra and O. Y. Abdallah, Novel skin penetrating berberine oleate complex capitalizing on hydrophobic ion pairing approach, Int. J. Pharm., 2018, 549(1-2), 76-86.
9 S. Bonengel, M. Jelkmann, M. Abdulkarim, M. Gumbleton, V. Reinstadler, H. Oberacher, F. Prufert and A. BernkopSchnurch, Impact of different hydrophobic ion pairs of octreotide on its oral bioavailability in pigs, $J$. Controlled Release, 2018, 273, 21-29.

10 M. Goswami, S. K. Kumar, A. Bhattacharya and J. F. Douglas, Computer Simulations of Ionomer SelfAssembly and Dynamics, Macromolecules, 2007, 40(12), 4113-4118.

11 J. Iqbal, C. Vigl, G. Moser, M. Gasteiger, G. Perera and A. Bernkop-Schnurch, Development and in vivo evaluation of a new oral nanoparticulate dosage form for leuprolide based on polyacrylic acid, Drug Delivery, 2011, 18(6), 432440.

12 R. F. Pagels and R. K. Prud'homme, Polymeric nanoparticles and microparticles for the delivery of peptides, biologics, and soluble therapeutics, J. Controlled Release, 2015, 219, 519-535.

13 R. Singh and J. W. Lillard Jr, Nanoparticle-based targeted drug delivery, Exp. Mol. Pathol., 2009, 86(3), 215-223.

14 C. E. Markwalter, R. F. Pagels, B. K. Wilson, K. D. Ristroph and R. K. Prud'homme, Flash NanoPrecipitation for the Encapsulation of Hydrophobic and Hydrophilic Compounds in Polymeric Nanoparticles, JoVE, 2019, (143), e58757.

15 K. T. Savjani, A. K. Gajjar and J. K. Savjani, Drug solubility: importance and enhancement techniques, ISRN Pharm., 2012, 2012, 195727.

16 S. Kumar, D. Bhargava, A. Thakkar and S. Arora, Drug carrier systems for solubility enhancement of BCS class II drugs: a critical review, Crit. Rev. Ther. Drug Carrier Syst., 2013, 30(3), 217-256.

17 C. Wischke and S. P. Schwendeman, Principles of encapsulating hydrophobic drugs in PLA/PLGA microparticles, Int. J. Pharm., 2008, 364(2), 298-327.

18 B. Devrim and A. S. Bozkir, in Design and Evaluation of Hydrophobic Ion-Pairing Complexation of Lysozyme with Sodium Dodecyl Sulfate for Improved Encapsulation of Hydrophilic Peptides/Proteins by Lipid-Polymer Hybrid Nanoparticles, 2015.

19 S. M. Ansell, S. A. Johnstone, P. G. Tardi, L. Lo, S. Xie, Y. Shu, T. O. Harasym, N. L. Harasym, L. Williams, D. Bermudes, B. D. Liboiron, W. Saad, R. K. Prud'homme and L. D. Mayer, Modulating the Therapeutic Activity of Nanoparticle Delivered Paclitaxel by Manipulating the Hydrophobicity of Prodrug Conjugates, J. Med. Chem., 2008, 51(11), 3288-3296.

20 J. S. Sohn, J. I. Jin, M. Hess and B. W. Jo, Polymer prodrug approaches applied to paclitaxel, Polym. Chem., 2010, 1(6), 778-792.

21 V. J. Stella, Prodrugs: Some thoughts and current issues, $J$. Pharm. Sci., 2010, 99(12), 4755-4765.

22 E. E. Dormidontova, Role of Competitive PEO-Water and Water-Water Hydrogen Bonding in Aqueous Solution PEO Behavior, Macromolecules, 2002, 35(3), 987-1001.

23 Y. Zhang, S. Furyk, D. E. Bergbreiter and P. S. Cremer, Specific Ion Effects on the Water Solubility of 
Macromolecules: PNIPAM and the Hofmeister Series, $J$. Am. Chem. Soc., 2005, 127(41), 14505-14510.

24 R. J. Goddard, B. P. Grady and S. L. Cooper, The Room Temperature Annealing Peak in Ionomers: Ionic Crystallites or Water Absorption?, Macromolecules, 1994, 27(7), 1710-1719.

25 B. A. Brozoski, P. C. Painter and M. M. Coleman, Concerning the origin of broad bands observed in the FTIR spectra of ionomers. Cluster formation or water adsorption?, Macromolecules, 1984, 17(8), 1591-1594.

26 J.-S. Kim and A. Eisenberg, Effect of sample preparation conditions and degree of neutralization on the dynamic mechanical properties of poly(styrene-co-sodium methacrylate) ionomers, J. Polym. Sci., Part B: Polym. Phys., 1995, 33(2), 197-209.

27 S. Yang, K. Sun and W. M. Risen Jr., Preparation and thermal characterization of the glass transition temperatures of sulfonated polystyrene-metal ionomers, $J$. Polym. Sci., Part B: Polym. Phys., 1990, 28(10), 1685-1697.

28 M. Morgen, A. Saxena, X. Q. Chen, W. Miller, R. Nkansah, A. Goodwin, J. Cape, R. Haskell, C. Su, O. Gudmundsson, M. Hageman, A. Kumar, G. S. Chowan, A. Rao and V. K. Holenarsipur, Lipophilic salts of poorly soluble compounds to enable high-dose lipidic SEDDS formulations in drug discovery, Eur. J. Pharm. Biopharm., 2017, 117, 212-223.

29 B. K. Poudel, B. Gupta, T. Ramasamy, R. K. Thapa, Y. S. Youn, H. G. Choi, C. S. Yong and J. O. Kim, Development of polymeric irinotecan nanoparticles using a novel lactone preservation strategy, Int. J. Pharm., 2016, 512(1), 75-86.

30 Z. Zhou, M. Jafari, V. Sriram, J. Kim and J. Y. Lee, Delayed Sequential Co-Delivery of Gefitinib and Doxorubicin for Targeted Combination, Chemotherapy, 2017, 14(12), 45514559.

31 P. R. Cullis and M. J. Hope, Lipid Nanoparticle Systems for Enabling Gene Therapies, Mol. Ther., 2017, 25(7), 14671475.

32 L. Xu and T. Anchordoquy, Drug delivery trends in clinical trials and translational medicine: Challenges and opportunities in the delivery of nucleic acid-based therapeutics, J. Pharm. Sci., 2011, 100(1), 38-52.

33 M. A. Mintzer and E. E. Simanek, Nonviral Vectors for Gene Delivery, Chem. Rev., 2009, 109(2), 259-302.

34 K. A. Whitehead, R. Langer and D. G. Anderson, Knocking down barriers: advances in siRNA delivery, Nat. Rev. Drug Discovery, 2009, 8(2), 129-138.

35 W. Li and F. C. Szoka, Lipid-based Nanoparticles for Nucleic Acid Delivery, Pharm. Res., 2007, 24(3), 438-449.

36 S. A. Wissing, O. Kayser and R. H. Muller, Solid lipid nanoparticles for parenteral drug delivery, Adv. Drug Delivery Rev., 2004, 56(9), 1257-1272.

37 W. Mehnert and K. Mader, Solid lipid nanoparticles: production, characterization and applications, Adv. Drug Delivery Rev., 2001, 47(2-3), 165-196.

38 Y. H. Song, E. Shin, H. Wang, J. Nolan, S. Low, D. Parsons, S. Zale, S. Ashton, M. Ashford, M. Ali, D. Thrasher,
N. Boylan and G. Troiano, A novel in situ hydrophobic ion paring (HIP) formulation strategy for clinical product selection of a nanoparticle drug delivery system, $J$. Controlled Release, 2016, 229, 106-119.

39 M. J. Heffernan, S. P. Kasturi, S. C. Yang, B. Pulendran and N. Murthy, The stimulation of CD8+ $\mathrm{T}$ cells by dendritic cells pulsed with polyketal microparticles containing ionpaired protein antigen and poly(inosinic acid)poly(cytidylic acid), Biomaterials, 2009, 30(5), 910-918.

40 T. Satoh, Y. Higuchi, S. Kawakami, M. Hashida, H. Kagechika, K. Shudo and M. Yokoyama, Encapsulation of the synthetic retinoids Am80 and LE540 into polymeric micelles and the retinoids' release control, J. Controlled Release, 2009, 136(3), 187-195.

41 I. Shahzadi, M. H. Asim, A. Dizdarević, J. D. Wolf, M. Kurpiers, B. Matuszczak and A. Bernkop-Schnürch, Arginine-based cationic surfactants: Biodegradable auxiliary agents for the formation of hydrophobic ion pairs with hydrophilic macromolecular drugs, J. Colloid Interface Sci., 2019, 552, 287-294.

42 T. Karamanidou, K. Karidi, V. Bourganis, K. Kontonikola, O. Kammona and C. Kiparissides, Effective incorporation of insulin in mucus permeating self-nanoemulsifying drug delivery systems, Eur. J. Pharm. Biopharm., 2015, 97(Pt A), 223-229.

43 H. D. Lu, K. D. Ristroph, E. L. K. Dobrijevic, J. Feng, S. A. McManus, Y. Zhang, W. D. Mulhearn, H. Ramachandruni, A. Patel and R. K. Prud'homme, Encapsulation of OZ439 into Nanoparticles for Supersaturated Drug Release in Oral Malaria Therapy, ACS Infect. Dis., 2018.

44 L. Feng, A. De Dille, V. J. Jameson, L. Smith, W. S. Dernell and M. C. Manning, Improved potency of cisplatin by hydrophobic ion pairing, Cancer Chemother. Pharmacol., 2004, 54(5), 441-448.

45 J. Chamieh, A. Domènech Tarrat, C. Doudou, V. Jannin, F. Demarne and H. Cottet, Peptide release from SEDDS containing hydrophobic ion pair therapeutic peptides measured by Taylor dispersion analysis, Int. J. Pharm., 2019, 559, 228-234.

46 G. A. Castro, A. L. Coelho, C. A. Oliveira, G. A. Mahecha, R. L. Orefice and L. A. Ferreira, Formation of ion pairing as an alternative to improve encapsulation and stability and to reduce skin irritation of retinoic acid loaded in solid lipid nanoparticles, Int. J. Pharm., 2009, 381(1), 77-83.

47 N. M. Pinkerton, A. Grandeury, A. Fisch, J. Brozio, B. U. Riebesehl and R. K. Prud'homme, Formation of stable nanocarriers by in situ ion pairing during blockcopolymer-directed rapid precipitation, Mol. Pharm., 2013, 10(1), 319-328.

48 K. Patel, V. Sarma and P. Vavia, Design and evaluation of lumefantrine - oleic acid self nanoemulsifying ionic complex for enhanced dissolution, Daru, J. Fac. Pharm., Tehran Univ. Med. Sci., 2013, 21(1), 27.

49 R. Bussano, D. Chirio, L. Costa, F. Turci and M. Trotta, Preparation and Characterization of Insulin-Loaded Lipid- 
Based Microspheres Generated by Electrospray, $J$. Dispersion Sci. Technol., 2011, 32(10), 1524-1530.

$50 \mathrm{~W}$. G. Dai and L. C. Dong, Characterization of physiochemical and biological properties of an insulin/ lauryl sulfate complex formed by hydrophobic ion pairing, Int. J. Pharm., 2007, 336(1), 58-66.

51 S. Sun, F. Cui, Y. Kawashima, N. Liang, L. Zhang, K. Shi and Y. Yu, A novel insulin-sodium oleate complex for oral administration: preparation, characterization and in vivo evaluation, J. Drug Delivery Sci. Technol., 2008, 18(4), 239243.

52 F. Cui, K. Shi, L. Zhang, A. Tao and Y. Kawashima, Biodegradable nanoparticles loaded with insulinphospholipid complex for oral delivery: preparation, in vitro characterization and in vivo evaluation, J. Controlled Release, 2006, 114(2), 242-250.

53 A. Elsayed, M. Al-Remawi, N. Qinna, A. Farouk, K. A. AlSou'od and A. A. Badwan, Chitosan-sodium lauryl sulfate nanoparticles as a carrier system for the in vivo delivery of oral insulin, AAPS PharmSciTech, 2011, 12(3), 958-964.

$54 \mathrm{~K}$. Talley and E. Alexov, On the $\mathrm{pH}$-optimum of activity and stability of proteins, Proteins, 2010, 78(12), 2699-2706.

55 D. Otzen, Protein-surfactant interactions: a tale of many states, Biochim. Biophys. Acta, 2011, 1814(5), 562-591.

56 K. Fu, R. Harrell, K. Zinski, C. Um, A. Jaklenec, J. Frazier, N. Lotan, P. Burke, A. M. Klibanov and R. Langer, A potential approach for decreasing the burst effect of protein from PLGA microspheres, J. Pharm. Sci., 2003, 92(8), 1582-1591.

57 H. S. Yoo, H. K. Choi and T. G. Park, Protein-fatty acid complex for enhanced loading and stability within biodegradable nanoparticles, J. Pharm. Sci., 2001, 90(2), 194-201.

58 U. Bilati, E. Allemann and E. Doelker, Nanoprecipitation versus emulsion-based techniques for the encapsulation of proteins into biodegradable nanoparticles and processrelated stability issues, AAPS PharmSciTech, 2005, 6(4), E594-E604.

59 R. Gaudana, M. Gokulgandhi, V. Khurana, D. Kwatra and A. K. Mitra, Design and evaluation of a novel nanoparticulate-based formulation encapsulating a HIP complex of lysozyme, Pharm. Dev. Technol., 2013, 18(3), 752-759.

60 H. Zhou, C. Lengsfeld, D. J. Claffey, J. A. Ruth, B. Hybertson, T. W. Randolph, K. Y. Ng and M. C. Manning, Hydrophobic ion pairing of isoniazid using a prodrug approach, J. Pharm. Sci., 2002, 91(6), 1502-1511.

61 H. L. Wong, R. Bendayan, A. M. Rauth and X. Y. Wu, Development of solid lipid nanoparticles containing ionically complexed chemotherapeutic drugs and chemosensitizers, J. Pharm. Sci., 2004, 93(8), 1993-2008.

62 Database., N. C. f. B. I. P. Sodium oleate, $C I D=23665730$. https://pubchem.ncbi.nlm.nih.gov/compound/23665730.

63 Database., N. C. f. B. I. P. Oleic acid, CID=445639. https:// pubchem.ncbi.nlm.nih.gov/compound/445639.

64 R. S. Kalhapure, C. Mocktar, D. R. Sikwal, S. J. Sonawane, M. K. Kathiravan, A. Skelton and T. Govender, Ion pairing with linoleic acid simultaneously enhances encapsulation efficiency and antibacterial activity of vancomycin in solid lipid nanoparticles, Colloids Surf., B, 2014, 117, 303-311.

65 G. Carneiro, E. L. Silva, L. A. Pacheco, E. M. de SouzaFagundes, N. C. Correa, A. M. de Goes, M. C. de Oliveira and L. A. Ferreira, Formation of ion pairing as an alternative to improve encapsulation and anticancer activity of all-trans retinoic acid loaded in solid lipid nanoparticles, Int. J. Nanomed., 2012, 7, 6011-6020.

66 W. Yin, Z. Dong, X. Chen, N. Finn and M. Z. Yates, Hydrophobic ion pairing to enhance encapsulation of water-soluble additives into $\mathrm{CO}_{2}$-swollen polymer microparticles, J. Supercrit. Fluids, 2007, 41(2), 293-298.

67 D. E. Kuehner, J. Engmann, F. Fergg, M. Wernick, H. W. Blanch and J. M. Prausnitz, Lysozyme Net Charge and Ion Binding in Concentrated Aqueous Electrolyte Solutions, J. Phys. Chem. B, 1999, 103(8), 1368-1374.

$68 \mathrm{~V}$. Spassov and L. Yan, A pH-dependent computational approach to the effect of mutations on protein stability, 2016, vol. 37.

69 G. Yu, J. Liu and J. Zhou, Mesoscopic coarse-grained simulations of hydrophobic charge induction chromatography (HCIC) for protein purification, AIChE J., 2015, 61(6), 2035-2047.

70 S. V. Mussi, R. C. Silva, M. C. Oliveira, C. M. Lucci, R. B. Azevedo and L. A. Ferreira, New approach to improve encapsulation and antitumor activity of doxorubicin loaded in solid lipid nanoparticles, Eur. J. Pharm. Sci., 2013, 48(1-2), 282-290.

71 Z. Zhou, C. Kennell, M. Jafari, J. Y. Lee, S. J. Ruiz-Torres, S. E. Waltz and J. H. Lee, Sequential delivery of erlotinib and doxorubicin for enhanced triple negative Breast cancer treatment using polymeric nanoparticle, Int. J. Pharm., 2017, 530(1-2), 300-307.

72 A. Siddiqui, V. Gupta, Y. Y. Liu and S. Nazzal, Doxorubicin and MBO-asGCS oligonucleotide loaded lipid nanoparticles overcome multidrug resistance in adriamycin resistant ovarian cancer cells (NCI/ADR-RES), Int. J. Pharm., 2012, 431(1-2), 222-229.

73 J. K. Guillory, Handbook of Pharmaceutical Salts: Properties, Selection, and Use Edited by P. Heinrich Stahl and Camile G. Wermuth. VHCA, Verlag Helvetica Chimica Acta, Zürich, Switzerland, and Wiley-VCH, Weinheim, Germany. 2002. vix +374 pp. $17.5 \times 24.5 \mathrm{~cm}$. ISBN 3906390-26-8. \$130.00, J. Med. Chem., 2003, 46(7), 1277.

$74 \mathrm{~W}$. Q. Tong and G. Whitesell, In situ salt screening-a useful technique for discovery support and preformulation studies, Pharm. Dev. Technol., 1998, 3(2), 215-223.

$75 \mathrm{I}$. Ghosh and W. M. Nau, The strategic use of supramolecular pKa shifts to enhance the bioavailability of drugs, Adv. Drug Delivery Rev., 2012, 64(9), 764-783.

76 N. i. Saleh, A. L. Koner and W. M. Nau, Activation and Stabilization of Drugs by Supramolecular pKa Shifts: Drug-Delivery Applications Tailored for Cucurbiturils, Angew. Chem., Int. Ed., 2008, 47(29), 5398-5401.

77 O. Zupancic, G. Leonaviciute, H. T. Lam, A. Partenhauser, S. Podricnik and A. Bernkop-Schnurch, Development and 
in vitro evaluation of an oral SEDDS for desmopressin, Drug Delivery, 2016, 23(6), 2074-2083.

78 H. D. Lu, P. Rummaneethorn, K. D. Ristroph and R. K. Prud'homme, Hydrophobic Ion Pairing of Peptide Antibiotics for Processing into Controlled Release Nanocarrier Formulations, Mol. Pharm., 2018, 15(1), 216225.

79 S. Zafar, L. M. Negi, A. K. Verma, V. Kumar, A. Tyagi, P. Singh, Z. Iqbal and S. Talegaonkar, Sterically stabilized polymeric nanoparticles with a combinatorial approach for multi drug resistant cancer: in vitro and in vivo investigations, Int. J. Pharm., 2014, 477(1-2), 454-468.

80 R. Rastogi, S. Anand and V. Koul, Evaluation of pharmacological efficacy of 'insulin-surfoplex' encapsulated polymer vesicles, Int. J. Pharm., 2009, 373(12), 107-115.

81 J. Fu, F. Sun, W. Liu, Y. Liu, M. Gedam, Q. Hu, C. Fridley, H. A. Quigley, J. Hanes and I. Pitha, Subconjunctival Delivery of Dorzolamide-Loaded Poly(ether-anhydride) Microparticles Produces Sustained Lowering of Intraocular Pressure in Rabbits, Mol. Pharm., 2016, 13(9), 2987-2995.

82 E. Imbuluzqueta, E. Elizondo, C. Gamazo, E. Moreno-Calvo, J. Veciana, N. Ventosa and M. J. Blanco-Prieto, Novel bioactive hydrophobic gentamicin carriers for the treatment of intracellular bacterial infections, Acta Biomater., 2011, 7(4), 1599-1608.

83 M. Gallarate, M. Trotta, L. Battaglia and D. Chirio, Cisplatin-loaded SLN produced by coacervation technique, J. Drug Delivery Sci. Technol., 2010, 20(5), 343347.

84 M. Gallarate, L. Battaglia, E. Peira and M. Trotta, PeptideLoaded Solid Lipid Nanoparticles Prepared through Coacervation Technique, Int. J. Chem. Eng., 2011, 2011, 6.

$85 \mathrm{H}$. Sang Yoo and T. Gwan Park, Biodegradable nanoparticles containing protein-fatty acid complexes for oral delivery of salmon calcitonin, J. Pharm. Sci., 2004, 93(2), 488-495.

86 J. Griesser, G. Hetenyi, M. Moser, F. Demarne, V. Jannin and A. Bernkop-Schnurch, Hydrophobic ion pairing: Key to highly payloaded self-emulsifying peptide drug delivery systems, Int. J. Pharm., 2017, 520(1-2), 267-274.

87 H. Yuan, S. P. Jiang, Y. Z. Du, J. Miao, X. G. Zhang and F. Q. Hu, Strategic approaches for improving entrapment of hydrophilic peptide drugs by lipid nanoparticles, Colloids Surf., B, 2009, 70(2), 248-253.

88 V. Jain, D. Singodia, G. K. Gupta, D. Garg, G. B. S. Keshava, R. Shukla, P. K. Shukla and P. R. Mishra, Ciprofloxacin surfplexes in sub-micron emulsions: A novel approach to improve payload efficiency and antimicrobial efficacy, Int. J. Pharm., 2011, 409(1), 237-244.

89 S. Ashton, Y. H. Song, J. Nolan, E. Cadogan, J. Murray, R. Odedra, J. Foster, P. A. Hall, S. Low, P. Taylor, R. Ellston, U. M. Polanska, J. Wilson, C. Howes, A. Smith, R. J. A. Goodwin, J. G. Swales, N. Strittmatter, Z. Takáts, A. Nilsson, P. Andren, D. Trueman, M. Walker, C. L. Reimer, G. Troiano, D. Parsons, D. De Witt,
M. Ashford, J. Hrkach, S. Zale, P. J. Jewsbury and S. T. Barry, Aurora kinase inhibitor nanoparticles target tumors with favorable therapeutic index in vivo, Sci. Transl. Med., 2016, 8(325), 325ra17.

90 A. Patel, R. Gaudana and A. K. Mitra, A novel approach for antibody nanocarriers development through hydrophobic ion-pairing complexation, J. Microencapsulation, 2014, 31(6), 542-550.

91 R. Gaudana, V. Khurana, A. Parenky and A. K. Mitra, Encapsulation of Protein-Polysaccharide HIP Complex in Polymeric Nanoparticles, J. Drug Delivery, 2011, 2011.

92 R. D. Vaishya, A. Mandal, M. Gokulgandhi, S. Patel and A. K. Mitra, Reversible hydrophobic ion-paring complex strategy to minimize acylation of octreotide during longterm delivery from PLGA microparticles, Int. J. Pharm., 2015, 489(1-2), 237-245.

93 S. Sun, N. Liang, Y. Kawashima, D. Xia and F. Cui, Hydrophobic ion pairing of an insulin-sodium deoxycholate complex for oral delivery of insulin, Int. J. Nanomed., 2011, 6, 3049-3056.

94 F. Hintzen, G. Perera, S. Hauptstein, C. Muller, F. Laffleur and A. Bernkop-Schnurch, In vivo evaluation of an oral self-microemulsifying drug delivery system (SMEDDS) for leuprorelin, Int. J. Pharm., 2014, 472(1-2), 20-26.

95 P. Ma, X. Dong, C. L. Swadley, A. Gupte, M. Leggas, H. C. Ledebur and R. J. Mumper, Development of idarubicin and doxorubicin solid lipid nanoparticles to overcome Pgp-mediated multiple drug resistance in leukemia, J. Biomed. Nanotechnol., 2009, 5(2), 151-161.

96 R. Mahjub, F. A. Dorkoosh, M. Rafiee-Tehrani and A. Bernkop Schnurch, Oral self-nanoemulsifying peptide drug delivery systems: impact of lipase on drug release, $J$. Microencapsulation, 2015, 32(4), 401-407.

97 C. Leichner, C. Menzel, F. Laffleur and A. BernkopSchnurch, Development and in vitro characterization of a papain loaded mucolytic self-emulsifying drug delivery system (SEDDS), Int. J. Pharm., 2017, 530(1-2), 346-353.

98 M. Ijaz, S. Bonengel, O. Zupancic, M. Yaqoob, M. Hartl, S. Hussain, C. W. Huck and A. Bernkop-Schnurch, Development of oral self nano-emulsifying delivery system(s) of lanreotide with improved stability against presystemic thiol-disulfide exchange reactions, Expert Opin. Drug Delivery, 2016, 13(7), 923-929.

99 A. Adjei, S. Rao, J. Garren, G. Menon and M. Vadnere, Effect of ion-pairing on 1-octanol-water partitioning of peptide drugs. I: the nonapeptide leuprolide acetate, Int. J. Pharm., 1993, 90(2), 141-149.

100 M. E. Powers, J. Matsuura, J. Brassell, M. C. Manning and E. Shefter, Enhanced solubility of proteins and peptides in nonpolar solvents through hydrophobic ion pairing, Biopolymers, 1993, 33(6), 927-932.

101 S. H. Choi and T. G. Park, Hydrophobic ion pair formation between leuprolide and sodium oleate for sustained release from biodegradable polymeric microspheres, Int. J. Pharm., 2000, 203(1-2), 193-202.

102 S. Sun, N. Liang, H. Piao, H. Yamamoto, Y. Kawashima and F. Cui, Insulin-S.O (sodium oleate) complex-loaded PLGA 
nanoparticles: formulation, characterization and in vivo evaluation, J. Microencapsulation, 2010, 27(6), 471-478.

103 H. M. Abdelaziz, A. O. Elzoghby, M. W. Helmy, M. W. Samaha, J. Y. Fang and M. S. Freag, Liquid crystalline assembly for potential combinatorial chemoherbal drug delivery to lung cancer cells, Int. J. Nanomed., 2019, 14, 499-517.

104 G. Dalwadi and B. Sunderland, An ion pairing approach to increase the loading of hydrophilic and lipophilic drugs into PEGylated PLGA nanoparticles, Eur. J. Pharm. Biopharm., 2009, 71(2), 231-242.

105 M. S. Oliveira, S. V. Mussi, D. A. Gomes, M. I. Yoshida, F. Frezard, V. M. Carregal and L. A. M. Ferreira, alphaTocopherol succinate improves encapsulation and anticancer activity of doxorubicin loaded in solid lipid nanoparticles, Colloids Surf., B, 2016, 140, 246-253.

106 X. Zhang, X. Sun, J. Li, X. Zhang, T. Gong and Z. Zhang, Lipid nanoemulsions loaded with doxorubicin-oleic acid ionic complex: characterization, in vitro and in vivo studies, Pharmazie, 2011, 66(7), 496-505.

107 G. A. Castro, R. L. Orefice, J. M. Vilela, M. S. Andrade and L. A. Ferreira, Development of a new solid lipid nanoparticle formulation containing retinoic acid for topical treatment of acne, J. Microencapsulation, 2007, 24(5), 395-407.

108 E. L. Silva, G. Carneiro, P. A. Caetano, G. Goulart, D. Ferreira Costa, E. M. de Souza-Fagundes, D. A. Gomes and L. A. Ferreira, Nanostructured lipid carriers loaded with tributyrin as an alternative to improve anticancer activity of all-trans retinoic acid, Expert Rev. Anticancer Ther., 2015, 15(2), 247-256.

109 L. Yang, F. Cui, K. Shi, D. Cun and R. Wang, Design of high payload PLGA nanoparticles containing melittin/sodium dodecyl sulfate complex by the hydrophobic ion-pairing technique, Drug Dev. Ind. Pharm., 2009, 35(8), 959-968.

110 C. Dumont, S. Bourgeois, H. Fessi, P. Y. Dugas and V. Jannin, In-vitro evaluation of solid lipid nanoparticles: Ability to encapsulate, release and ensure effective protection of peptides in the gastrointestinal tract, Int. J. Pharm., 2019, 565, 409-418.

111 K. Shi, F. Cui, H. Yamamoto and Y. Kawashima, Investigation of drug loading and in vitro release mechanisms of insulin-lauryl sulfate complex loaded PLGA nanoparticles, Pharmazie, 2008, 63(12), 866-871.

112 H. Xu, L. Zhang, L. Li, Y. Liu, Y. Chao, X. Liu, Z. Jin, Y. Chen, X. Tang, H. He, Q. Kan and C. Cai, Membrane-Loaded Doxorubicin Liposomes Based on Ion-Pairing Technology with High Drug Loading and $\mathrm{pH}$-Responsive Property, AAPS PharmSciTech, 2017, 18(6), 2120-2130.

113 H. D. Lu, K. D. Ristroph, E. L. K. Dobrijevic, J. Feng, S. A. McManus, Y. Zhang, W. D. Mulhearn, H. Ramachandruni, A. Patel and R. K. Prud'homme, Encapsulation of OZ439 into Nanoparticles for Supersaturated Drug Release in Oral Malaria Therapy, ACS Infect. Dis., 2018, 4(6), 970-979.

114 G. A. Castro, L. A. M. Ferreira, R. L. Oréfice and V. T. L. Buono, Characterization of a new solid lipid nanoparticle formulation containing retinoic acid for topical treatment of acne, Powder Diffr., 2012, 23(S1), S30S35.

115 E. L. Silva, F. A. Lima, G. Carneiro, P. Ramos Jonas, D. A. Gomes, E. M. de Souza-Fagundes and L. A. Ferreira, Improved In Vitro Antileukemic Activity of All-Trans Retinoic Acid Loaded in Cholesteryl Butyrate Solid Lipid Nanoparticles, J. Nanosci. Nanotechnol., 2016, 16(2), 12911300.

116 L. Battaglia, M. Gallarate, E. Peira, D. Chirio, E. Muntoni, E. Biasibetti, M. T. Capucchio, A. Valazza, P. P. Panciani, M. Lanotte, D. Schiffer, L. Annovazzi, V. Caldera, M. Mellai and C. Riganti, Solid lipid nanoparticles for potential doxorubicin delivery in glioblastoma treatment: preliminary in vitro studies, J. Pharm. Sci., 2014, 103(7), 2157-2165.

117 R. Falk, T. W Randolph, J. D Meyer, R. M. Kelly and M. Manning, Controlled release of ionic compounds from poly (L-lactide) microspheres produced by precipitation with a compressed antisolvent, 1997, vol. 44, pp. 77-85.

118 E. Elizondo, S. Sala, E. Imbuluzqueta, D. Gonzalez, M. J. Blanco-Prieto, C. Gamazo, N. Ventosa and J. Veciana, High loading of gentamicin in bioadhesive PVM/MA nanostructured microparticles using compressed carbon-dioxide, Pharm. Res., 2011, 28(2), 309321.

119 E. Imbuluzqueta, S. Lemaire, C. Gamazo, E. Elizondo, N. Ventosa, J. Veciana, F. Van Bambeke and M. J. BlancoPrieto, Cellular pharmacokinetics and intracellular activity against Listeria monocytogenes and Staphylococcus aureus of chemically modified and nanoencapsulated gentamicin, J. Antimicrob. Chemother., 2012, 67(9), 21582164.

120 R. Gaudana, A. Parenky, R. Vaishya, S. K. Samanta and A. K. Mitra, Development and characterization of nanoparticulate formulation of a water soluble prodrug of dexamethasone by HIP complexation, $J$. Microencapsulation, 2011, 28(1), 10-20.

121 S. Zhao, L. V. Minh, N. Li, V. M. Garamus, U. A. Handge, J. Liu, R. Zhang, R. Willumeit-Romer and A. Zou, Doxorubicin hydrochloride-oleic acid conjugate loaded nanostructured lipid carriers for tumor specific drug release, Colloids Surf., B, 2016, 145, 95-103.

122 K. Fu, D. W. Pack, A. M. Klibanov and R. Langer, Visual Evidence of Acidic Environment Within Degrading Poly(lactic-co-glycolic acid) (PLGA) Microspheres, Pharm. Res., 2000, 17(1), 100-106.

123 E. D. Goddard, Polymer/surfactant interaction-Its relevance to detergent systems, J. Am. Oil Chem. Soc., 1994, 71(1), 1-16.

124 J. T. G. Overbeek and M. J. Voorn, Phase separation in polyelectrolyte solutions. Theory of complex coacervation, J. Cell. Comp. Physiol., 1957, 49(S1), 7-26.

125 J. v. d. Gucht, E. Spruijt, M. Lemmers and M. A. Cohen Stuart, Polyelectrolyte complexes: Bulk phases and colloidal systems, J. Colloid Interface Sci., 2011, 361(2), 407-422. 
126 N. R. Johnson and Y. Wang, Coacervate delivery systems for proteins and small molecule drugs, Expert Opin. Drug Delivery, 2014, 11(12), 1829-1832.

127 J. Lee, Y. O. Popov and G. H. Fredrickson, Complex coacervation: A field theoretic simulation study of polyelectrolyte complexation, J. Chem. Phys., 2008, 128(22), 224908.

128 K. T. Delaney and G. H. Fredrickson, Theory of polyelectrolyte complexation-complex coacervates are self-coacervates, J. Chem. Phys., 2017, 146(22), 224902.

129 P. Pincus, Colloid stabilization with grafted polyelectrolytes, Macromolecules, 1991, 24(10), 2912-2919.

130 Y. O. Popov, J. Lee and G. H. Fredrickson, Field-theoretic simulations of polyelectrolyte complexation, J. Polym. Sci., Part B: Polym. Phys., 2007, 45(24), 3223-3230.

131 M. Muthukumar, 50th Anniversary Perspective: A Perspective on Polyelectrolyte Solutions, Macromolecules, 2017, 50(24), 9528-9560.

132 P. Guenoun, H. T. Davis, M. Tirrell and J. W. Mays, Aqueous Micellar Solutions of Hydrophobically Modified Polyelectrolytes, Macromolecules, 1996, 29(11), 3965-3969.

133 Q. Wang and J. B. Schlenoff, The Polyelectrolyte Complex/ Coacervate Continuum, Macromolecules, 2014, 47(9), 3108-3116.

134 W. Xiong, C. Ren, W. Jin, J. Tian, Y. Wang, B. R. Shah, J. Li and B. Li, Ovalbumin-chitosan complex coacervation: Phase behavior, thermodynamic and rheological properties, Food Hydrocolloids, 2016, 61, 895-902.

135 Y. Zhang, F. Li, L. D. Valenzuela, M. Sammalkorpi and J. L. Lutkenhaus, Effect of Water on the Thermal Transition Observed in Poly(allylamine hydrochloride)Poly(acrylic acid) Complexes, Macromolecules, 2016, 49(19), 7563-7570.

136 H. K. Awada, N. R. Johnson and Y. Wang, Dual Delivery of Vascular Endothelial Growth Factor and Hepatocyte Growth Factor Coacervate Displays Strong Angiogenic Effects, Macromol. Biosci., 2014, 14(5), 679-686.

137 H. Bysell, P. Hansson and M. Malmsten, Effect of Charge Density on the Interaction between Cationic Peptides and Oppositely Charged Microgels, J. Phys. Chem. B, 2010, 114(21), 7207-7215.

138 A. Nolles, A. H. Westphal, J. A. de Hoop, R. G. Fokkink, J. M. Kleijn, W. J. H. van Berkel and J. W. Borst, Encapsulation of GFP in Complex Coacervate Core Micelles, Biomacromolecules, 2015, 16(5), 1542-1549.

139 C. B. Packhaeuser and T. Kissel, On the design of in situ forming biodegradable parenteral depot systems based on insulin loaded dialkylaminoalkyl-amine-poly(vinyl alcohol)-g-poly(lactide-co-glycolide) nanoparticles, $J$. Controlled Release, 2007, 123(2), 131-140.

140 A. Obermeyer, E. C. Mills, X.-H. Dong, R. J. Flores and B. Olsen, Complex coacervation of supercharged proteins with polyelectrolytes, 2016, vol. 12.

141 K. A. Black, D. Priftis, S. L. Perry, J. Yip, W. Y. Byun and M. Tirrell, Protein Encapsulation via Polypeptide Complex Coacervation, ACS Macro Lett., 2014, 3(10), 1088-1091.
142 M. N. Jones, The interaction of sodium dodecyl sulfate with polyethylene oxide, J. Colloid Interface Sci., 1967, 23(1), 3642.

143 E. D. Goddard and R. B. Hannan, Cationic polymer/anionic surfactant interactions, J. Colloid Interface Sci., 1976, 55(1), 73-79.

144 R. Y. Lochhead and L. R. Huisinga, A brief review of polymer/ surfactant interaction, Feb 2004.

145 M. S. Johal and P. A. Chiarelli, Polymer-surfactant complexation in polyelectrolyte multilayer assemblies, Soft Matter, 2007, 3(1), 34-46.

146 E. D. Goddard and R. B. Hannan, Polymer/surfactant interactions, J. Am. Oil Chem. Soc., 1977, 54(12), 561-566.

147 M. Goswami, J. M. Borreguero, P. A. Pincus and B. G. Sumpter, Surfactant-Mediated Polyelectrolyte SelfAssembly in a Polyelectrolyte-Surfactant Complex, Macromolecules, 2015, 48(24), 9050-9059.

148 Y. Wang, K. Kimura, Q. Huang, P. L. Dubin and W. Jaeger, Effects of Salt on Polyelectrolyte-Micelle Coacervation, Macromolecules, 1999, 32(21), 7128-7134.

149 M. Manuszak Guerrini, R. Lochhead and W. Daly, Interactions of aminoalkylcarbamoyl cellulose derivatives and sodium dodecyl sulfate. 2. Foam stabilization, 1999, vol. 147, pp. 67-78.

150 M. Antonietti, J. Conrad and A. Thuenemann, Polyelectrolyte-Surfactant Complexes: A New Type of Solid, Mesomorphous Material, Macromolecules, 1994, 27(21), 6007-6011.

151 J. Fundin and W. Brown, Polymer/Surfactant Interactions. Sodium Poly(styrene sulfonate) and CTAB Complex Formation. Light Scattering Measurements in Dilute Aqueous Solution, Macromolecules, 1994, 27(18), 50245031.

152 Â. M. L. Denadai, A. M. de Oliveira, I. M. P. Daniel, L. A. Carneiro, K. C. Ribeiro, H. d. O. Beraldo, K. J. R. da Costa, V. C. da Cunha, M. E. Cortés and R. D. Sinisterra, Chlorhexidine/losartan ionic pair binding and its nanoprecipitation: physico-chemical characterisation and antimicrobial activity, Supramol. Chem., 2012, 24(3), 204212.

153 W. Li, X. Yi, X. Liu, Z. Zhang, Y. Fu and T. Gong, Hyaluronic acid ion-pairing nanoparticles for targeted tumor therapy, J. Controlled Release, 2016, 225, 170-182.

154 K. D. Ristroph, J. Feng, S. A. McManus, Y. Zhang, K. Gong, H. Ramachandruni, C. E. White and R. K. Prud'homme, Spray drying OZ439 nanoparticles to form stable, waterdispersible powders for oral malaria therapy, J. Transl. Med., 2019, $17(1), 97$.

155 I. Lozoya-Agullo, M. Planelles, M. Merino-Sanjuán, M. Bermejo, B. Sarmento, I. González-Álvarez and M. González-Álvarez, Ion-pair approach coupled with nanoparticle formation to increase bioavailability of a low permeability charged drug, Int. J. Pharm., 2019, 557, 36-42.

156 C. Tang, E. Zhang, Y. Li and L. Yang, An innovative method for preparation of hydrophobic ion-pairing colistin entrapped poly(lactic acid) nanoparticles: Loading and 
release mechanism study, Eur. J. Pharm. Sci., 2017, 102, 6370 .

157 J. Liu, T. Gong, C. Wang, Z. Zhong and Z. Zhang, Solid lipid nanoparticles loaded with insulin by sodium cholatephosphatidylcholine-based mixed micelles: preparation and characterization, Int. J. Pharm., 2007, 340(1-2), 153162.

158 G. P. Zara, R. Cavalli, A. Fundaro, A. Bargoni, O. Caputo and M. R. Gasco, Pharmacokinetics of doxorubicin incorporated in solid lipid nanospheres (SLN), Pharmacol. Res., 1999, 40(3), 281-286.

159 S. Morel, E. Ugazio, R. Cavalli and M. R. Gasco, Thymopentin in solid lipid nanoparticles, Int. J. Pharm., 1996, 132(1), 259-261.

160 R. Cavalli, M. R. Gasco, P. Chetoni, S. Burgalassi and M. F. Saettone, Solid lipid nanoparticles (SLN) as ocular delivery system for tobramycin, Int. J. Pharm., 2002, 238(1-2), 241-245.

161 M. S. Freag, A. S. Torky, M. M. Nasra, D. A. Abdelmonsif and O. Y. Abdallah, Liquid crystalline nanoreservoir releasing a highly skin-penetrating berberine oleate complex for psoriasis management, Nanomedicine, 2019, 14(8), 931954.

162 S. Sun, N. Liang, H. Yamamoto, Y. Kawashima, F. Cui and P. Yan, pH-sensitive poly(lactide-co-glycolide) nanoparticle composite microcapsules for oral delivery of insulin, Int. J. Nanomed., 2015, 10, 3489-3498.

163 H. Zhao, H. Lu, T. Gong and Z. Zhang, Nanoemulsion loaded with lycobetaine-oleic acid ionic complex: physicochemical characteristics, in vitro, in vivo evaluation, and antitumor activity, Int. J. Nanomed., 2013, 8, 1959-1973.

164 T. Zhang, Y. Zheng, Q. Peng, X. Cao, T. Gong and Z. Zhang, A novel submicron emulsion system loaded with vincristine-oleic acid ion-pair complex with improved anticancer effect: in vitro and in vivo studies, Int. J. Nanomed., 2013, 8, 1185-1196.

165 I. Nazir, M. H. Asim, A. Dizdarevic and A. BernkopSchnurch, Self-emulsifying drug delivery systems: Impact of stability of hydrophobic ion pairs on drug release, Int. J. Pharm., 2019, 561, 197-205.

166 N. Mittapelly, M. Thalla, G. Pandey, V. T. Banala, S. Sharma, A. Arya, S. Mishra, K. Mitra, S. Shukla and P. R. Mishra, Long Acting Ionically Paired Embonate Based Nanocrystals of Donepezil for the Treatment of Alzheimer's Disease: a Proof of Concept Study, Pharm. Res., 2017, 34(11), 2322-2335.

167 S. J. Novick and J. S. Dordick, Protein-containing hydrophobic coatings and films, Biomaterials, 2002, 23(2), 441-448.

168 L. Battaglia, M. Gallarate, E. Peira, D. Chirio, I. Solazzi, S. Marzia Adele Giordano, C. Gigliotti, C. Riganti and
C. Dianzani, Bevacizumab loaded solid lipid nanoparticles prepared by the coacervation technique: Preliminary in vitro studies. 2015, vol. 26, p. 255102.

169 N. Lupo, V. N. Tkadlečková, M. Jelkmann, F. Laffleur, G. Hetényi, K. Kubová and A. Bernkop-Schnürch, Selfemulsifying drug delivery systems: In vivo evaluation of their potential for oral vaccination, Acta Biomater., 2019.

170 J. Griesser, G. Hetenyi, H. Kadas, F. Demarne, V. Jannin and A. Bernkop-Schnurch, Self-emulsifying peptide drug delivery systems: How to make them highly mucus permeating, Int. J. Pharm., 2018, 538(1-2), 159-166.

171 R. Falk and T. W. Randolph, Process Variable Implications for Residual Solvent Removal and Polymer Morphology in the Formation of Gentamycin-Loaded Poly (L-lactide) Microparticles. 1998; vol. 15, pp. 1233-7.

172 R. Alcock, J. A. Blair, D. J. O'Mahony, A. Raoof and A. V. Quirk, Modifying the release of leuprolide from spray dried OED microparticles, J. Controlled Release, 2002, 82(2-3), 429-440.

173 A. D. Holmkvist, A. Friberg, U. J. Nilsson and J. Schouenborg, Hydrophobic ion pairing of a minocycline/ $\mathrm{Ca}^{2+} /$ AOT complex for preparation of drugloaded PLGA nanoparticles with improved sustained release, Int. J. Pharm., 2016, 499(1), 351-357.

174 J. T. Evans, J. R. Ward, J. Kern and M. E. Johnson, A single vaccination with protein-microspheres elicits a strong CD8 T-cell-mediated immune response against Mycobacterium tuberculosis antigen Mtb8.4, Vaccine, 2004, 22(15-16), 1964-1972.

175 M. Hill, R. N. Cunningham, R. M. Hathout, C. Johnston, J. G. Hardy and M. E. Migaud, Formulation of Antimicrobial Tobramycin Loaded PLGA Nanoparticles via Complexation with AOT, J. Funct. Biomater., 2019, 10(2), 26.

176 N. A. Efiana, A. Dizdarevic, C. W. Huck and A. BernkopSchnurch, Improved intestinal mucus permeation of vancomycin via incorporation into nanocarrier containing papain-palmitate, J. Pharm. Sci., 2019.

177 K. Shi, F. Cui, H. Yamamoto and Y. Kawashima, Optimized preparation of insulin-lauryl sulfate complex loaded poly (lactide-co-glycolide) nanoparticles using response surface methodology, Pharmazie, 2008, 63(10), 721-725.

178 K. Shi, F. Cui, H. Yamamoto and Y. Kawashima, Optimized formulation of high-payload PLGA nanoparticles containing insulin-lauryl sulfate complex, Drug Dev. Ind. Pharm., 2009, 35(2), 177-184.

179 H. Zhou, Y. Zhang, D. L. Biggs, M. C. Manning, T. W. Randolph, U. Christians, B. M. Hybertson and K. Y. Ng, Microparticle-based lung delivery of INH decreases INH metabolism and targets alveolar macrophages, J. Controlled Release, 2005, 107(2), 288-299. 